\title{
One-loop analysis of the interactions between charmed mesons and Goldstone bosons
}

\author{
De-Liang Yao, ${ }^{a}$ Meng-Lin Du, ${ }^{b}$ Feng-Kun Guo ${ }^{c, b}$ and UIf-G. Meißner ${ }^{b, a}$ \\ ${ }^{a}$ Institute for Advanced Simulation, Institut für Kernphysik and \\ Jülich Center for Hadron Physics, Forschungszentrum Jülich, \\ Wilhelm-Johnen-Straße, D-52425 Jülich, Germany \\ ${ }^{b}$ Helmholtz-Institut für Strahlen- und Kernphysik and \\ Bethe Center for Theoretical Physics, Universität Bonn, \\ Nußallee 14-16, D-53115 Bonn, Germany \\ c State Key Laboratory of Theoretical Physics, \\ Institute of Theoretical Physics, Chinese Academy of Science, \\ Zhong Guan Cun East Street 55, Beijing 100190, China \\ E-mail: d.yao@fz-juelich.de, du@hiskp.uni-bonn.de, fkguo@itp.ac.cn, \\ meissner@hiskp.uni-bonn.de
}

ABSTRACT: We derive the scattering amplitude for Goldstone bosons of chiral symmetry off the pseudoscalar charmed mesons up to leading one-loop order in a covariant chiral effective field theory, using the so-called extended-on-mass-shell renormalization scheme. Then we use unitarized chiral perturbation theory to fit to the available lattice data of the $S$-wave scattering lengths. The lattice data are well described. However, most of the low-energy constants determined from the fit bear large uncertainties. Lattice simulations in more channels are necessary to pin down these values which can then be used to make predictions in other processes related by chiral and heavy quark symmetries.

KEYWords: QCD Phenomenology

ArXiv EPrint: 1502.05981 


\section{Contents}

1 Introduction 1

2 Theoretical framework $\quad 4$

2.1 Power counting and power counting breaking terms 4

2.2 Chiral effective Lagrangian 5

$3 \quad D$ - $\phi$ scattering amplitudes up to NNLO $\quad 6$

3.1 Tree-level contribution $\quad 6$

3.2 One-loop contribution $\quad 8$

3.3 Renormalization 8

3.3.1 Wave function renormalization 9

$\begin{array}{lll}\text { 3.3.2 Extended-on-mass-shell subtraction scheme } & 10\end{array}$

4 Partial wave amplitudes and unitarization $\quad 11$

$\begin{array}{lll}4.1 & \text { Amplitudes for given strangeness and isospin } & 12\end{array}$

$\begin{array}{lll}4.2 & \text { Partial wave projection } & 12\end{array}$

$\begin{array}{lll}4.3 & \text { Unitarization } & 13\end{array}$

5 Calculation of the scattering lengths $\quad \mathbf{1 5}$

$\begin{array}{ll}5.1 \text { Definition and pion mass dependence } & 15\end{array}$

$\begin{array}{lll}5.2 & \text { Fits to lattice data on the scattering lengths } & 16\end{array}$

$\begin{array}{lll}\text { 5.2.1 Introduction to the fitting procedure } & 16\end{array}$

$\begin{array}{lll}5.2 .2 & \text { Results } & 18\end{array}$

5.2.3 Contribution of vector charmed mesons 24

6 Summary and outlook 26

$\begin{array}{ll}\text { A Definition of one-loop integrals } & 27\end{array}$

$\begin{array}{ll}\text { B Loop amplitudes without explicit charmed vector mensons } & 28\end{array}$

C Infrared regular parts of the loop integrals $\quad 30$

\section{Introduction}

The hadronic interaction between charmed $D$ mesons and the Goldstone bosons $\phi$ of the spontaneous breaking of chiral symmetry of the strong interaction $(D-\phi$ interaction for short hereafter) is important for the understanding of the chiral dynamics of quantum chromodynamics (QCD) and the interpretation of the hadron spectrum in the heavy hadron sector. Many investigations have been devoted to study it in the last decade, partly triggered by 
the observation of the charm-strange meson $D_{s 0}^{*}(2317)$ with $J^{P}=0^{+}$in $2003[1,2]$. The $D_{s 0}^{*}(2317)$ couples to the $D K$ channel, and being below the $D K$ threshold it decays into the isospin breaking channel $D_{s} \pi$. In order to unravel its nature, theorists study the $D-\phi$ interaction and intend to extract the information encoded in it. For instance, the $D_{s 0}^{*}(2317)$ is interpreted as a $D K$ molecule [3] by using a chiral unitary approach to the $S$-wave $D$ - $\phi$ interaction [4-6]. In these works, the leading order (LO) amplitudes from the heavy meson chiral perturbation theory (ChPT) [7-9] are used as the kernels of resummed amplitudes. Extensions to the next-to-leading order (NLO) can be found in refs. [10-14].

Recently, renewed interest was stimulated due to the occurrence of lattice QCD calculations of the scattering lengths given in refs. [15, 16]. In these works, only channels free of disconnected Wick contractions are calculated, which are $D \pi$ with isospin $I=3 / 2$, $D \bar{K}$ with $I=0$ and $1, D_{s} K$ and $D_{s} \pi$. There have been lattice results on channels with disconnected Wick contractions, such as $D \pi$ with $I=1 / 2$ [17] and $D K$ with $I=0$ [18]. With these lattice calculations, more insights were gained into the nature of the $D_{s 0}^{*}(2317)$. The $D K$ isoscalar scattering length was calculated indirectly in ref. [16], which is consistent with the result from the direct lattice calculation in refs. [18, 19]. A reanalysis of the lattice energy levels for the $D^{(*)} K$ lattice data [19] was performed in ref. [20] in terms of an auxiliary potential and an extended Lüscher formula. These results suggests that the $D_{s 0}^{*}(2317)$ is dominantly a $D K$ hadronic molecule. ${ }^{1}$

The lattice data can be used to determine the low-energy constants (LECs) in the chiral Lagrangian of higher orders. Especially, the lattice data in refs. [15, 16] were used in refs. [12, 16, 23-27]. In the majority of those investigations, unitarized extensions of ChPT, see e.g. refs. [28, 29], are adopted so that one can consider larger meson masses and channel couplings. The unitarized chiral perturbation theory (UChPT) is especially necessary for the chiral extrapolation of scattering lengths in question since for larger quark (or meson) masses the interaction normally becomes stronger and could even be nonperturbative. However, in all the calculations in the framework of unitarized ChPT, the kernel of the resummed amplitude was only calculated up to NLO at most and is purely tree-level. Here, we will extend the calculation to the leading one-loop order, which is the next-to-next-to-leading order (NNLO).

It is well-known that ChPT [30-32] has become an useful and standard tool in studying the hadron interaction at low energies. Based on Weinberg's power counting rules [30], great achievements have been obtained both in the pure mesonic sector and the one including matter fields such as baryons, the latter known as baryon ChPT. There is a notable power counting breaking (PCB) issue in baryon ChPT [33]: using the dimensional regularization with the modified minimal subtraction $(\overline{\mathrm{MS}})$ scheme in calculating loop integrals, the naive power counting does not work and all loop diagrams start contributing at $\mathcal{O}\left(p^{2}\right)$, with $p$ being a small momentum. There have been several solutions to this problem: heavy baryon (HB) approach [34, 35], infrared regularizaion (IR) [36] and extended-on-mass-shell (EOMS) scheme [37] (for a review and a detailed comparison of these approaches, see ref. [38]).

\footnotetext{
${ }^{1} \mathrm{~A}$ method of extracting the probability of a physical state to be a hadronic molecule in lattice using twisted boundary conditions is discussed in ref. [21] where the $D_{s 0}^{*}(2317)$ is used as an example for illustration. The $D_{s 0}^{*}(2317)$ was also studied in a finite volume in ref. [22].
} 
Likewise, ChPT including the heavy $D$ mesons encounters the same PCB problem. To remedy it, in ref. [23], the $D$ - $\phi$ scattering lengths were calculated in the framework of nonrelativistic heavy meson ChPT [7-9] to the leading one-loop order in the heavy quark limit. Nevertheless, as mentioned by the authors and confirmed by ref. [24], this nonrelavistic formulation neglects sizable recoil corrections. ${ }^{2}$ The calculations in various unitarized versions of ChPT in refs. [12, 16, 25, 26] are performed in a covariant formalism, but only up to NLO as mentioned above. The first NNLO calculation of the scattering lengths was given by ref. [24] using the EOMS scheme. However, the calculation in that work is perturbative while the interactions in certain channels are definitely nonperturbative. For instance, in the channel with $(S, I)=(1,0)$, where $S$ and $I$ represent strangeness and isospin, respectively, the existence of the $D_{s 0}^{*}(2317)$ below the $D K$ threshold calls for a nonperturbative treatment of the $D K$ interaction or inclusion of an explicit field for the $D_{s 0}^{*}(2317)$. In addition, all the NNLO counterterm contributions are neglected in ref. [24] due to the poorly known LECs. In this paper, we intend to present a detailed covariant description of the $D-\phi$ interaction up to NNLO in the framework of UChPT, and the EOMS approach which preserves the proper analytic structure of the amplitudes will be used in renormalization procedure.

First, we will calculate the $D$ - $\phi$ scattering amplitude in covariant ChPT up to the NNLO. To our knowledge, the $D$ - $\phi$ scattering amplitudes (without vector charmed mesons) shown in the present work are the first analytical and complete results up to NNLO. ${ }^{3}$ The vector charmed meson contributions, surviving in the heavy quark limit, are also taken into account numerically to estimate their influences, although it was shown in ref. [13] that their contribution to the $S$-wave scattering is small. Renormalization will be performed using the EOMS scheme and it will be shown explicitly that the UV divergences are cancelled properly and PCB terms are absorbed exactly by the counterterms, which ensures that the EOMS-renormalized $D$ - $\phi$ scattering amplitudes possess the proper analytic, power counting, and scale-independent properties.

We will fix the values of the LECs by fitting to the available lattice data of the $S$-wave $D$ - $\phi$ scattering lengths. Since the lattice calculations are performed at large unphysical quark masses, the perturbative expansion to a certain order may fail to converge. One way to solve this issue is to employ unitarized amplitudes instead of the perturbative ones. Many unitarization methods have been proposed in the past. In ref. [29], a unitarization approach is developed and used to study the $\bar{K} N$ interaction. The unitarized amplitude can be matched to the perturbative amplitude order by order. Throughout this paper, we will call this approach UChPT for convenience. In refs. [40-44], the inverse amplitude method (IAM) was proposed and adopted to study the $\pi \pi$ and $K \pi$ scattering. For the purpose of comparison, both the two approaches will be employed.

\footnotetext{
${ }^{2}$ It is, however, known since a long time that in the heavy baryon approach such recoil corrections can easily be incorporated by using as the propagator $i /\left(v \cdot l-l^{2} / 2 m\right)$ instead of simply $i / v \cdot l[39]$.

${ }^{3}$ The analytical expressions for the amplitudes involving vector charmed mesons, which survive in the heavy quark limit, are too lengthy to be shown explicitly in the paper and can be made available upon request from the authors.
} 
This paper is organized as follows. In section 2, the power counting and and its breaking by heavy meson masses will be explained briefly and the chiral effective Lagrangian will be given up to NNLO. In section 3 , details on the computation of the $D$ - $\phi$ scattering amplitude using the EOMS scheme are exhibited. Together with the loop results shown in appendix B, the minimal but complete set of scattering amplitudes are given explicitly. Section 4 discusses how to obtain the partial wave amplitudes with definite strangeness $S$ and isospin $I$ from the physical process amplitudes, and the two unitarization approaches mentioned above will be introduced. In section 5 , the $S$-wave scattering lengths are calculated and fitted to the available lattice data at a few values of the pion masses, and the contributions of the vector charmed mesons will also be discussed. Finally, section 6 comprises a summary and outlook. Some technicalities are relegated to the appendices.

\section{Theoretical framework}

\subsection{Power counting and power counting breaking terms}

We denote the $D$ - $\phi$ interaction as $D_{1}\left(p_{1}\right) \phi_{1}\left(p_{2}\right) \rightarrow D_{2}\left(p_{3}\right) \phi_{2}\left(p_{4}\right)$. The scattering process is on-shell, hence, $p_{1}^{2}=M_{D_{1}}^{2}, p_{2}^{2}=M_{\phi_{1}}^{2}, p_{3}^{2}=M_{D_{2}}^{2}$ and $p_{4}^{2}=M_{\phi_{2}}^{2}$, with $M_{D_{1}}\left(M_{\phi_{1}}\right)$ and $M_{D_{2}}\left(M_{\phi_{2}}\right)$ being the masses of the incoming and outgoing $D$ mesons (Goldstone bosons), respectively. In addition, the Mandelstam variables are defined as

$$
s=\left(p_{1}+p_{2}\right)^{2}, \quad t=\left(p_{1}-p_{3}\right)^{2}, \quad u=\left(p_{1}-p_{4}\right)^{2},
$$

which satisfy the relation $s+t+u=M_{D_{1}}^{2}+M_{\phi_{1}}^{2}+M_{D_{2}}^{2}+M_{\phi_{2}}^{2}$. At low energies, one has

$$
\frac{s-M_{D_{1}}^{2}}{\Lambda_{\chi}^{2}} \sim \frac{s-M_{D_{2}}^{2}}{\Lambda_{\chi}^{2}} \sim \frac{u-M_{D_{1}}^{2}}{\Lambda_{\chi}^{2}} \sim \frac{u-M_{D_{2}}^{2}}{\Lambda_{\chi}^{2}} \sim \frac{M_{\phi_{1}}}{\Lambda_{\chi}} \sim \frac{M_{\phi_{2}}}{\Lambda_{\chi}} \ll 1, \quad \frac{t}{\Lambda_{\chi}^{2}} \ll 1,
$$

where $\Lambda_{\chi} \sim\left\{4 \pi F_{\pi}, M_{D_{1}}, M_{D_{2}}\right\}$ denotes the high energy scale, with $F_{\pi}$ the pion decay constant $F_{\pi} \simeq 92.2 \mathrm{MeV}$. The above small quantities can be simultaneously adopted as expansion parameters. In a more conventional notation, one denotes the small parameters by a unique symbol, say $p$, so that the power counting rules for the basic quantities read

$$
\begin{aligned}
& M_{D_{1}} \sim O\left(p^{0}\right), \quad M_{D_{2}} \sim O\left(p^{0}\right), \quad M_{\phi_{1}} \sim O\left(p^{1}\right), \quad M_{\phi_{2}} \sim O\left(p^{1}\right), \quad t \sim O\left(p^{2}\right), \\
& s-M_{D_{1}}^{2} \sim O\left(p^{1}\right), \quad s-M_{D_{2}}^{2} \sim O\left(p^{1}\right), \quad u-M_{D_{1}}^{2} \sim O\left(p^{1}\right), \quad u-M_{D_{2}}^{2} \sim O\left(p^{1}\right) .
\end{aligned}
$$

It is worth noting that the the chiral limit masses of the charmed mesons are of the same order as the corresponding physical masses. Every physical observable therefore has its own chiral dimension by using the above given power counting rules.

Furthermore, in ChPT a power counting rule is assigned for each Feynman graph. In the present case, the chiral dimension $n$ for a given graph can be evaluated from

$$
n=4 L+\sum_{k} V_{k}-2 I_{\phi}-I_{D}
$$

where $L, V_{k}, I_{\phi}$ and $I_{D}$ are the numbers of loops, $k^{\text {th }}$ order vertices, Goldstone boson propagators and charmed meson propagators, respectively. 
For a specific physical observable, if there exist terms with chiral dimensions obtained using eq. (2.3) lower than that given by eq. (2.4), those terms are called PCB terms. The PCB terms show up only when there are heavy particles with nonvanishing chiral limit masses in loops as internal propagators. In our present calculation, since the heavy charmed mesons are involved in some of one-loop graphs, there will be PCB terms if we use dimensional regularization with the $\overline{\mathrm{MS}}$ scheme. These terms can be treated in the so-called EOMS scheme, which has a power counting consistent with eq. (2.4), as will be detailed in section 3 .

\subsection{Chiral effective Lagrangian}

The pseudoscalar charmed mesons can be collected in a $\mathrm{SU}(3)$ triplet, $D=\left(D^{0}, D^{+}, D_{s}^{+}\right)$, and the light Goldstone bosons are in an octet,

$$
\phi=\left(\begin{array}{ccc}
\frac{1}{\sqrt{2}} \pi^{0}+\frac{1}{\sqrt{6}} \eta & \pi^{+} & K^{+} \\
\pi^{-} & -\frac{1}{\sqrt{2}} \pi^{0}+\frac{1}{\sqrt{6}} \eta & K^{0} \\
K^{-} & \bar{K}^{0} & -\frac{2}{\sqrt{6}} \eta
\end{array}\right) .
$$

The chiral effective Lagrangian for $D$ - $\phi$ scattering can be decomposed into $D$ mesonGoldstone boson interacting parts and pure Goldstone bosonic parts, which has the following form:

$$
\mathcal{L}_{\text {eff }}=\mathcal{L}_{D \phi}^{(1)}+\mathcal{L}_{D \phi}^{(2)}+\mathcal{L}_{D \phi}^{(3)}+\mathcal{L}_{\phi \phi}^{(2)}+\mathcal{L}_{\phi \phi}^{(4)}+\ldots
$$

Here, the numbers in the superscripts stand for the chiral dimensions, and the ellipsis denotes the higher-order chiral operators which will not be used here. Besides, the operators with external fields are also dropped (except for the scalar external field which is used for the light quark mass insertions).

The familiar lowest order chiral Lagrangian for the Goldstone boson sector reads

$$
\mathcal{L}_{\phi \phi}^{(2)}=\frac{F_{0}^{2}}{4}\left\langle\partial_{\mu} U\left(\partial^{\mu} U\right)^{\dagger}\right\rangle+\frac{F_{0}^{2}}{4}\left\langle\chi U^{\dagger}+U \chi^{\dagger}\right\rangle,
$$

with $U=\exp \left(i \sqrt{2} \phi / F_{0}\right)$ and $\chi=2 B_{0} \operatorname{diag}\left(m_{u}, m_{d}, m_{s}\right)$. Here $\langle\ldots\rangle$ denotes the trace in the light-flavor space, $F_{0}$ is the pion decay constant in the chiral limit, and $B_{0}$ is a constant related to the quark condensate. We will work in the isospin limit with $m_{u}=m_{d}$ and neglect the electromagnetic contributions.

The $O\left(p^{4}\right)$ pure Goldstone boson Lagrangian $\mathcal{L}_{\phi \phi}^{(4)}$ is needed for renormalization. Its LECs enter the $D-\phi$ amplitudes merely through the wave renormalization constants and the decay constants of the Goldstone bosons, which can be found elsewhere, see e.g. ref. [32]. The relevant terms read

$$
\mathcal{L}_{\phi \phi}^{(4)}=L_{4}\left\langle\partial_{\mu} U\left(\partial^{\mu} U\right)^{\dagger}\right\rangle\left\langle\chi U^{\dagger}+U \chi^{\dagger}\right\rangle+L_{5}\left\langle\partial_{\mu} U\left(\partial^{\mu} U\right)^{\dagger}\left(\chi U^{\dagger}+U \chi^{\dagger}\right)\right\rangle+\ldots
$$

For the interaction in the $D$ meson-Goldstone boson sector, the LO effective Lagrangian takes the form

$$
\mathcal{L}_{D \phi}^{(1)}=\mathcal{D}_{\mu} D \mathcal{D}^{\mu} D^{\dagger}-M_{0}^{2} D D^{\dagger},
$$


where $M_{0}$ is the mass of the $D$ mesons in the chiral limit, and the covariant derivative acting on the $D$ mesons is defined by

$$
\mathcal{D}_{\mu} D=D\left(\overleftarrow{\partial_{\mu}}+\Gamma_{\mu}^{\dagger}\right), \quad \mathcal{D}_{\mu} D^{\dagger}=\left(\partial_{\mu}+\Gamma_{\mu}\right) D^{\dagger}
$$

with the so-called chiral connection $\Gamma_{\mu}=\left(u^{\dagger} \partial_{\mu} u+u \partial_{\mu} u^{\dagger}\right) / 2$. The NLO Lagrangian reads $[11]^{4}$

$$
\begin{aligned}
\mathcal{L}_{D \phi}^{(2)}= & D\left(-h_{0}\left\langle\chi_{+}\right\rangle-h_{1} \chi_{+}+h_{2}\left\langle u_{\mu} u^{\mu}\right\rangle-h_{3} u_{\mu} u^{\mu}\right) D^{\dagger} \\
& +\mathcal{D}_{\mu} D\left(h_{4}\left\langle u_{\mu} u^{\nu}\right\rangle-h_{5}\left\{u^{\mu}, u^{\nu}\right\}\right) \mathcal{D}_{\nu} D^{\dagger}
\end{aligned}
$$

where the building blocks of the chiral effective Lagrangian are given by

$$
u_{\mu}=i\left(u^{\dagger} \partial_{\mu} u-u \partial_{\mu} u^{\dagger}\right), \quad u=\exp \left(\frac{i \phi}{\sqrt{2} F_{0}}\right), \quad \chi_{ \pm}=u^{\dagger} \chi u^{\dagger} \pm u \chi u
$$

Here, the definition for $\chi_{-}$is also given as it is needed for the NNLO Lagrangian which, following the procedure detailed in ref. [45], can be constructed as

$$
\begin{aligned}
\mathcal{L}_{D \phi}^{(3)}= & D\left[i g_{1}\left[\chi_{-}, u_{\nu}\right]+g_{2}\left(\left[u_{\mu},\left[\mathcal{D}_{\nu}, u^{\mu}\right]\right]+\left[u_{\mu},\left[\mathcal{D}^{\mu}, u_{\nu}\right]\right]\right)\right] \mathcal{D}^{\nu} D^{\dagger} \\
& +g_{3} D\left[u_{\mu},\left[\mathcal{D}_{\nu}, u_{\rho}\right]\right] \mathcal{D}^{\mu \nu \rho} D^{\dagger}
\end{aligned}
$$

where the totally symmetrized product of three covariant derivatives is defined as $\mathcal{D}^{\mu \nu \rho}=$ $\left\{\mathcal{D}_{\mu},\left\{\mathcal{D}_{\nu}, \mathcal{D}_{\rho}\right\}\right\}$.

\section{$3 \quad D$ - $\phi$ scattering amplitudes up to NNLO}

In this section, we exhibit the complete set of independent $D$ - $\phi$ scattering amplitudes on the basis of the physical states. They correspond to 10 physical processes as listed in the second column in table 1 . All the other amplitudes can be obtained by using either crossing symmetry or time-reversal invariance. In what follows, we will first calculate the tree-level amplitude which can be reduced into a common structure but with different coefficients because of SU(3) flavor symmetry. Then the loop amplitudes will be given explicitly. In the end, the renormalization procedure within the EOMS scheme will be discussed.

\subsection{Tree-level contribution}

The Feynman diagrams of the tree-level contribution to the scattering amplitudes are displayed in the first line of figure 1. Since we do not consider the exchange of resonances,

\footnotetext{
${ }^{4}$ As in ref. [16], the $h_{6}$ term in ref. [11] is dropped, and the $\tilde{\chi}_{+}=\chi_{+}-\left\langle\chi_{+}\right\rangle / 3$ is replaced by $\chi_{+}$which amounts to a redefinition of $h_{0}$ and $h_{1}$. The $h_{6}$ term is redundant, since$$
-h_{6} \mathcal{D}_{\mu} D\left[u^{\mu}, u^{\nu}\right] \mathcal{D}_{\nu} D^{\dagger}=\frac{h_{6}}{2}\left\{D\left[u^{\mu}, u^{\nu}\right]\left(\mathcal{D}_{\mu} \mathcal{D}_{\nu} D^{\dagger}\right)+\left(\mathcal{D}_{\nu} \mathcal{D}_{\mu} D\right)\left[u^{\mu}, u^{\nu}\right] D^{\dagger}\right\}+\text { higher order terms },
$$

where the first term is zero due to the symmetry property of the Lorentz indices $\mu, \nu$, and the higher order terms are contained in the higher order Lagrangians.
} 


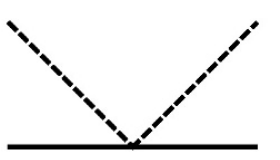

Tree (1)

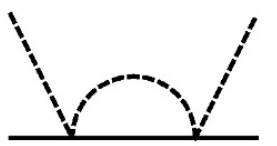

Loop (1)

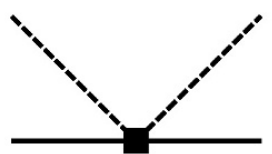

Tree (2)

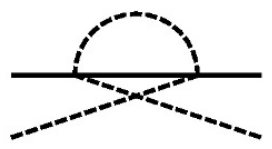

Loop (2)

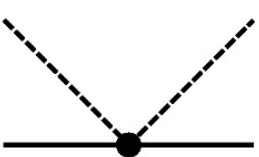

Tree (3)

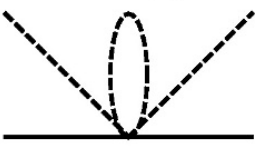

Loop (3)

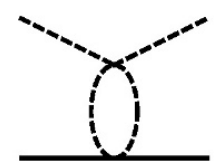

Loop (4)

Figure 1. The 1-point irreducible (1PI) Feynman diagrams for $D$ - $\phi$ scattering up to leading oneloop order. The solid (dashed) lines represent the $D$ (Goldstone) mesons. The square stands for the contact vertex coming from Lagrangian $\mathcal{L}_{D \phi}^{(2)}$, while the filled circle denotes an insertion from $\mathcal{L}_{D \phi}^{(3)}$. All other vertices are generated either by $\mathcal{L}_{D \phi}^{(1)}$ or $\mathcal{L}_{\phi \phi}^{(2)}$.

such contributions are encoded in the contact terms for the $D-\phi$ scattering. When calculating the Feynman diagrams, all the bare parameters, such as the decay constant $F_{0}$ and the masses, are maintained. They will be replaced by the corresponding physical quantities when the renormalization is performed. The LO, i.e. $\mathcal{O}(p)$, tree amplitude is the Weinberg-Tomozawa term, ${ }^{5}$ and has the following form,

$$
\mathcal{A}^{(1)}(s, t, u)=\mathcal{C}_{\mathrm{LO}} \frac{s-u}{4 F_{0}^{2}},
$$

where the coefficients $\mathcal{C}_{\mathrm{LO}}$ for different physical processes are listed in table 1 . The Weinberg-Tomozawa term depends only on the pion decay constant due to the fact that it originates from the kinetic term in $\mathcal{L}_{D \phi}^{(1)}$, which is a result of the spontaneous breaking of chiral symmetry in QCD.

The $\mathcal{O}\left(p^{2}\right)$ Lagrangian $\mathcal{L}_{D \phi}^{(2)}$ generates the tree-level contribution at NLO as

$$
\mathcal{A}^{(2)}(s, t, u)=\frac{1}{F_{0}^{2}}\left[-4 h_{0} \mathcal{C}_{0}^{(2)}+2 h_{1} \mathcal{C}_{1}^{(2)}-2 \mathcal{C}_{24}^{(2)} H_{24}(s, t, u)+2 \mathcal{C}_{35}^{(2)} H_{35}(s, t, u)\right],
$$

where the coefficients are shown in table 1 , and the functions $H_{24}(s, t, u)$ and $H_{35}(s, t, u)$ are defined by

$$
\begin{aligned}
& H_{24}(s, t, u)=2 h_{2} p_{2} \cdot p_{4}+h_{4}\left(p_{1} \cdot p_{2} p_{3} \cdot p_{4}+p_{1} \cdot p_{4} p_{2} \cdot p_{3}\right) \\
& H_{35}(s, t, u)=h_{3} p_{2} \cdot p_{4}+h_{5}\left(p_{1} \cdot p_{2} p_{3} \cdot p_{4}+p_{1} \cdot p_{4} p_{2} \cdot p_{3}\right)
\end{aligned}
$$

Finally, the tree-level amplitude at $\mathcal{O}\left(p^{3}\right)$ reads

$$
\mathcal{A}^{(3)}(s, t, u)=\frac{1}{F_{0}^{2}}\left\{4 g_{1}\left[\mathcal{C}_{1 a}^{(3)}\left(p_{1}+p_{3}\right) \cdot\left(p_{2}+p_{4}\right)+\mathcal{C}_{1 b}^{(3)}\left(p_{1}+p_{3}\right) \cdot p_{2}\right]+4 \mathcal{C}_{23}^{(3)} G_{23}(s, t, u)\right\},
$$

\footnotetext{
${ }^{5}$ As the vector charmed mesons are not taken into account, there is no Born term due to the exchange of these mesons.
} 


\begin{tabular}{|cl|c|cccc|}
\hline & Physical processes & $\mathcal{C}_{\mathrm{LO}}$ & $\mathcal{C}_{0}^{(2)}$ & $\mathcal{C}_{1}^{(2)}$ & $\mathcal{C}_{24}^{(2)}$ & $\mathcal{C}_{35}^{(2)}$ \\
\hline 1 & $D^{0} K^{-} \rightarrow D^{0} K^{-}$ & 1 & $M_{K}^{2}$ & $-M_{K}^{2}$ & 1 & 1 \\
2 & $D^{+} K^{+} \rightarrow D^{+} K^{+}$ & 0 & $M_{K}^{2}$ & 0 & 1 & 0 \\
3 & $D^{+} \pi^{+} \rightarrow D^{+} \pi^{+}$ & 1 & $M_{\pi}^{2}$ & $-M_{\pi}^{2}$ & 1 & 1 \\
4 & $D^{+} \eta \rightarrow D^{+} \eta$ & 0 & $M_{\eta}^{2}$ & $-\frac{1}{3} M_{\pi}^{2}$ & 1 & $\frac{1}{3}$ \\
5 & $D_{s}^{+} K^{+} \rightarrow D_{s}^{+} K^{+}$ & 1 & $M_{K}^{2}$ & $-M_{K}^{2}$ & 1 & 1 \\
6 & $D_{s}^{+} \eta \rightarrow D_{s}^{+} \eta$ & 0 & $M_{\eta}^{2}$ & $\frac{4}{3}\left(M_{\pi}^{2}-2 M_{K}^{2}\right)$ & 1 & $\frac{4}{3}$ \\
7 & $D_{s}^{+} \pi^{0} \rightarrow D_{s}^{+} \pi^{0}$ & 0 & $M_{\pi}^{2}$ & 0 & 1 & 0 \\
8 & $D^{0} \eta \rightarrow D^{0} \pi^{0}$ & 0 & 0 & $-\frac{1}{\sqrt{3}} M_{\pi}^{2}$ & 0 & $\frac{1}{\sqrt{3}}$ \\
9 & $D_{s}^{+} K^{-} \rightarrow D^{0} \pi^{0}$ & $-\frac{1}{\sqrt{2}}$ & 0 & $-\frac{1}{2 \sqrt{2}}\left(M_{K}^{2}+M_{\pi}^{2}\right)$ & 0 & $\frac{1}{\sqrt{2}}$ \\
10 & $D_{s}^{+} K^{-} \rightarrow D^{0} \eta$ & $-\sqrt{\frac{3}{2}}$ & 0 & $\frac{1}{2 \sqrt{6}}\left(5 M_{K}^{2}-3 M_{\pi}^{2}\right)$ & 0 & $-\frac{1}{\sqrt{6}}$ \\
\hline
\end{tabular}

Table 1. The coefficients in the LO and NLO tree-level amplitudes of the 10 relevant physical processes. The Gell-Mann-Okubo mass relation, $3 M_{\eta}^{2}=4 M_{K}^{2}-M_{\pi}^{2}$, is used to simplify the coefficients when necessary.

with

$$
\begin{aligned}
G_{23}(s, t, u)= & -g_{2} p_{2} \cdot p_{4}\left(p_{1}+p_{3}\right) \cdot\left(p_{2}+p_{4}\right) \\
& +2 g_{3}\left[\left(p_{1} \cdot p_{2}\right)\left(p_{1} \cdot p_{4}\right) p_{1} \cdot\left(p_{2}+p_{4}\right)+\left(p_{1} \rightarrow p_{3}\right)\right] .
\end{aligned}
$$

The corresponding coefficients can be found in table 2. The $\mathcal{C}_{1 b}^{(3)}$ term survives only for inelastic scattering processes.

\subsection{One-loop contribution}

The one-loop connected graphs for $D$ - $\phi$ scattering are shown in the second line of figure 1 . All the vertices in the loop graphs originate from the Lagrangians $\mathcal{L}_{D \phi}^{(1)}$ and $\mathcal{L}_{\phi \phi}^{(2)}$ which are free of unknown LECs. Similar to the tree-level amplitudes, it suffices to calculate the loop amplitudes for the 10 physical processes. All these loop amplitudes are listed in appendix B, which are expressed in terms of a set of one-loop integrals given in appendix A.

\subsection{Renormalization}

In the previous sections, the 1PI Feynman graphs are all calculated, which are related to the so-called amputated amplitudes. To derive the $S$-matrix elements, one should perform wave function renormalization. Moreover, in the end, all the bare parameters should be replaced by the corresponding physical ones. 


\begin{tabular}{|ll|ccc|}
\hline & physical process & $\mathcal{C}_{1 a}^{(3)}$ & $\mathcal{C}_{1 b}^{(3)}$ & $\mathcal{C}_{23}^{(3)}$ \\
\hline 1 & $D^{0} K^{-} \rightarrow D^{0} K^{-}$ & $M_{K}^{2}$ & 0 & 1 \\
2 & $D^{+} K^{+} \rightarrow D^{+} K^{+}$ & 0 & 0 & 0 \\
3 & $D^{+} \pi^{+} \rightarrow D^{+} \pi^{+}$ & $M_{\pi}^{2}$ & 0 & 1 \\
4 & $D^{+} \eta \rightarrow D^{+} \eta$ & 0 & 0 & 0 \\
5 & $D_{s}^{+} K^{+} \rightarrow D_{s}^{+} K^{+}$ & $M_{K}^{2}$ & 0 & 1 \\
6 & $D_{s}^{+} \eta \rightarrow D_{s}^{+} \eta$ & 0 & 0 & 0 \\
7 & $D_{s}^{+} \pi^{0} \rightarrow D_{s}^{+} \pi^{0}$ & 0 & 0 & 0 \\
8 & $D^{0} \eta \rightarrow D^{0} \pi^{0}$ & 0 & 0 & 0 \\
9 & $D_{s}^{+} K^{-} \rightarrow D^{0} \pi^{0}$ & $-\frac{1}{\sqrt{2}} M_{K}^{2}$ & $\frac{1}{\sqrt{2}}\left(M_{K}^{2}-M_{\pi}^{2}\right)$ & $-\frac{1}{\sqrt{2}}$ \\
10 & $D_{s}^{+} K^{-} \rightarrow D^{0} \eta$ & $-\sqrt{\frac{3}{2}} M_{K}^{2}$ & $\frac{1}{\sqrt{6}}\left(M_{\pi}^{2}-M_{K}^{2}\right)$ & $-\sqrt{\frac{3}{2}}$ \\
\hline
\end{tabular}

Table 2. The coefficients in the NNLO tree-level amplitudes of the 10 relevant physical processes.

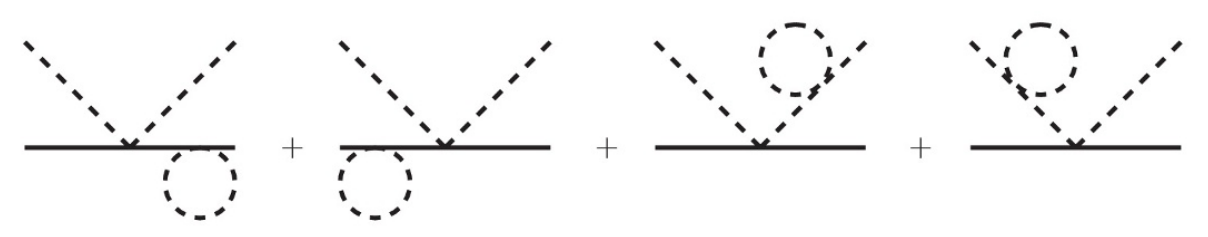

Figure 2. Feynman diagrams for the wave function renormalization at $\mathcal{O}\left(p^{3}\right)$.

\subsubsection{Wave function renormalization}

To perform the wave function renormalization, one multiplies the external lines with the square roots of the wave function renormalization constants of the corresponding fields and takes them on the mass shell. In perturbation theory, if the calculation is done up to a certain order (up to $\mathcal{O}\left(p^{3}\right)$ in our case), the wave function renormalization is equivalent to taking the graphs in figure 2 into account. All the higher order contributions beyond the required accuracy are ignored.

Hence, when taking wave function renormalization into consideration, the scattering amplitude becomes

$$
\mathcal{A}(s, t)=\mathcal{A}_{\text {tree }}^{(1)}(s, t)+\mathcal{A}_{\text {tree }}^{(2)}(s, t)+\mathcal{A}_{\text {tree }}^{(3)}(s, t)+\mathcal{A}_{\text {loop }}^{(3)}(s, t)+\mathcal{A}_{\mathrm{wf}}^{(3)}(s, t) .
$$

The first three terms are tree contribution given in section 3.1, while the fourth term is the loop contribution discussed in section 3.2 and appendix B. The last term $\mathcal{A}_{\mathrm{wf}}(s, t)$ corresponds to the contribution from the wave function renormalization. It can be obtained 
from the LO amplitude in combination with the wave function renormalization constants. For instance, considering the scattering process $D_{1} \phi_{1} \rightarrow D_{2} \phi_{2}$, it is given by

$$
\mathcal{A}_{\mathrm{wf}}^{(3)}(s, t)=\frac{1}{2}\left(\delta \mathcal{Z}_{D_{1}}+\delta \mathcal{Z}_{\phi_{1}}+\delta \mathcal{Z}_{D_{2}}+\delta \mathcal{Z}_{\phi_{2}}\right) \mathcal{A}_{\text {tree }}^{(1)}(s, t),
$$

with $\delta \mathcal{Z}=\mathcal{Z}-1$ and $\mathcal{Z}$ being the wave function renormalization constant up to the order considered. To be explicit, the wave function renormalization constants for $D$ and $D_{s}$ are $\mathcal{Z}_{D}=\mathcal{Z}_{D_{s}}=1$ and for the Goldstone bosons are

$$
\begin{aligned}
\mathcal{Z}_{\pi} & =1-\frac{1}{F_{0}^{2}}\left[8 L_{4}\left(2 M_{K}^{2}+M_{\pi}^{2}\right)+8 L_{5} M_{\pi}^{2}+\frac{1}{3} \mathcal{I}_{K}+\frac{2}{3} \mathcal{I}_{\pi}\right], \\
\mathcal{Z}_{K} & =1-\frac{1}{F_{0}^{2}}\left[8 L_{4}\left(2 M_{K}^{2}+M_{\pi}^{2}\right)+8 L_{5} M_{K}^{2}+\frac{1}{2} \mathcal{I}_{K}+\frac{1}{4} \mathcal{I}_{\pi}+\frac{1}{4} \mathcal{I}_{\eta}\right], \\
\mathcal{Z}_{\eta} & =1-\frac{1}{F_{0}^{2}}\left[8 L_{4}\left(2 M_{K}^{2}+M_{\pi}^{2}\right)+\frac{4}{3} L_{5}\left(4 M_{K}^{2}-M_{\pi}^{2}\right)+\mathcal{I}_{K}\right],
\end{aligned}
$$

where the tadpole loop integral $\mathcal{I}_{i}(i=\pi, K, \eta)$ can be found in appendix A. Note that in the above expressions, the ultraviolet (UV) divergence of the loop functions is not subtracted on purpose. This is due to the fact that the $\mathcal{Z}$ 's are not physical observables such that they might be divergent, namely the LECs $L_{4}$ and $L_{5}$ are not sufficient to absorb the UV divergence in those expressions. The UV divergence cancellation as well as the PCB terms absorption will be discussed in the following section at the level of the $S$-matrix elements. As one will see, the $S$-matrix elements are free of any divergence.

\subsubsection{Extended-on-mass-shell subtraction scheme}

The loop integrals in the amplitude shown in eq. (3.7) is UV divergent, and we need renormalization to absorb the divergences by counterterms. Moreover, PCB terms show up in the chiral expansion if we use dimensional regularization with the $\overline{\mathrm{MS}}$ scheme. It is necessary to get rid of them to have a good power counting. We will use the EOMS subtraction scheme which has the proper analyticity and correct power counting for the amplitudes. The essence of the EOMS scheme is to perform two subsequent subtractions: the $\overline{\mathrm{MS}}$ subtraction and the EOMS finite subtraction.

In the $\overline{\mathrm{MS}}$ subtraction, the UV divergent parts are extracted and then cancelled by the divergences in the bare LECs, which are separated into finite and divergent parts as follows:

$$
h_{i}=h_{i}^{r}(\mu)+\frac{\alpha_{i}}{16 \pi^{2} F_{0}^{2}} R, \quad g_{j}=g_{j}^{r}(\mu)+\frac{\beta_{j}}{16 \pi^{2} F_{0}^{2}} R, \quad L_{k}=L_{k}^{r}(\mu)+\frac{\Gamma_{k}}{32 \pi^{2}} R,
$$

where $R=\frac{2}{d-4}+\gamma_{E}-1-\ln (4 \pi)$ with $\gamma_{E}$ being the Euler constant, and $d$ is the space-time dimension. The coefficients $\alpha_{i}(i=0, \cdots, 5), \beta_{j}(j=1,2,3)$ and $\Gamma_{k}(k=4,5)$ are given by

$$
\begin{aligned}
& \alpha_{0}=0, \quad \alpha_{1}=0, \quad \alpha_{2}=\frac{M_{0}^{2}}{48}, \quad \alpha_{3}=-\frac{M_{0}^{2}}{16}, \quad \alpha_{4}=\frac{7}{24}, \quad \alpha_{5}=-\frac{7}{16}, \\
& \beta_{1}=0, \quad \beta_{2}=-\frac{9}{128}, \quad \beta_{3}=0, \quad \Gamma_{4}=\frac{1}{8}, \quad \Gamma_{5}=\frac{3}{8} \text {. }
\end{aligned}
$$


Although the UV divergences have been removed so far, it is still not sufficient to get an amplitude that respects the power counting rule given by eq. (2.4). The charmed mesons show up in the Feynman diagrams, say Loop(1) and Loop(2) in figure 1, and generate the so-called PCB terms that often spoil the convergence of the chiral expansion [33]. The EOMS finite subtraction is used to get rid of those PCB terms. For each physical process given above, the PCB terms are easily obtained by replacing the loop function $\mathcal{F}_{a b}^{(c d)}(s, t)$, see eq. (B.1), by $\mathcal{F}_{a b}^{(c d)}(s, t)^{\mathrm{PCB}}$, namely eq. (C.3), in the amplitudes and then performing the chiral expansion with respect to the small quantities. Note that the infrared regular parts for the required scalar loop integrals are also listed in appendix $\mathrm{C}$ for easy reference. Eventually, the PCB terms are absorbed by decomposing the $\overline{\mathrm{MS}}$-renormalized LECs in the $\mathcal{O}\left(p^{2}\right)$ Lagrangian via

$$
h_{i}^{r}(\mu)=\tilde{h}_{i}+\frac{\delta_{i}}{16 \pi^{2} F_{0}^{2}} M_{0}^{2},
$$

with the coefficient $\delta_{i}(i=0, \cdots, 5)$ defined by

$$
\begin{aligned}
& \delta_{0}=\delta_{1}=0, \quad \delta_{2}=-\frac{1}{72}+\frac{1}{48} \log \frac{M_{0}^{2}}{\mu^{2}}, \quad \delta_{3}=\frac{1}{24}-\frac{1}{16} \log \frac{M_{0}^{2}}{\mu^{2}}, \\
& \delta_{4}=-\frac{35}{72 M_{0}^{2}}+\frac{7}{24 M_{0}^{2}} \log \frac{M_{0}^{2}}{\mu^{2}}, \quad \delta_{5}=\frac{35}{48 M_{0}^{2}}-\frac{7}{16 M_{0}^{2}} \log \frac{M_{0}^{2}}{\mu^{2}} .
\end{aligned}
$$

The other LECs such as $g_{j}^{r}(\mu)$ and $L_{k}^{r}(\mu)$ are untouched when performing the finite EOMS subtraction.

After the two steps described above, we have obtained the full renormalized amplitudes. For the sake of easy practical usage, the chiral-limit $D$ meson mass $M_{0}$ and the chirallimit decay constant $F_{0}$ should be further related to the corresponding physical quantities according to the following expressions:

$$
\begin{aligned}
M_{D}^{2} & =M_{0}^{2}+2\left(h_{0}+h_{1}\right) M_{\pi}^{2}+4 h_{0} M_{K}^{2} \\
M_{D_{s}}^{2} & =M_{0}^{2}+2\left(h_{0}-h_{1}\right) M_{\pi}^{2}+4\left(h_{0}+h_{1}\right) M_{K}^{2}, \\
F_{\pi} & =F_{0}+\frac{1}{2 F_{0}}\left(2 \mathcal{I}_{\pi}^{r}+\mathcal{I}_{K}^{r}\right)+\frac{4 M_{\pi}^{2}}{F_{0}}\left(L_{4}^{r}+L_{5}^{r}\right)+\frac{8 M_{K}^{2}}{F_{0}} L_{4}^{r} .
\end{aligned}
$$

Here, we rewrite $F_{0}$ in terms of $F_{\pi}$ rather than $F_{K}$ and $F_{\eta}$. This is the convention to be used throughout. Alternatively, one can also rewrites it in terms of $F_{K}$ or $F_{\eta}$, and the difference is of higher order. The loop functions and LECs with a superscript $r$ stand for their finite parts, namely, the contributions proportional to the UV divergence $R$ are removed.

\section{Partial wave amplitudes and unitarization}

In this section, we will illustrate how to obtain partial wave amplitudes with definite strangeness $S$ and isospin $I$ from the 10 physical process amplitudes exhibited in the previous section in detail. Then we will discuss the unitarization of the scattering amplitudes using two different approaches. Based on the content of this section, it is straightforward to derive the $S$-wave scattering lengths, which will be discussed and compared with lattice data in the next section. 


\subsection{Amplitudes for given strangeness and isospin}

The scattering amplitudes in the isospin basis can be classified by two quantum numbers, which are the strangeness $S$ and isospin $I$ of the scattering system. Hereafter, the scattering amplitudes with definite strangeness and isospin are called strangeness-isospin amplitudes for short. All the strangeness-isospin amplitudes can be related to the 10 amplitudes of the physical processes using crossing symmetry and isospin symmetry.

We begin with the single-channel interactions. There are 4 single channels in total. The corresponding quantum numbers of $(S, I)$ are $(-1,0),(-1,1),(0,3 / 2)$ and $(2,1 / 2)$. Their strangeness-isospin amplitudes are related to the physical-process amplitudes by

$$
\begin{aligned}
\mathcal{A}_{D \bar{K} \rightarrow D \bar{K}}^{(-1,0)}(s, t, u) & =2 \mathcal{A}_{D^{+} K^{+} \rightarrow D^{+} K^{+}}(u, t, s)-\mathcal{A}_{D^{0} K^{-} \rightarrow D^{0} K^{-}}(s, t, u), \\
\mathcal{A}_{D \bar{K} \rightarrow D \bar{K}}^{(-1,1)}(s, t, u) & =\mathcal{A}_{D^{0} K^{-} \rightarrow D^{0} K^{-}}(s, t, u), \\
\mathcal{A}_{D \pi \rightarrow D \pi}^{(0,3 / 2)}(s, t, u) & =\mathcal{A}_{D^{+} \pi^{+} \rightarrow D^{+} \pi^{+}}(s, t, u), \\
\mathcal{A}_{D_{s} K \rightarrow D_{s} K}^{(2,1 / 2)}(s, t, u) & =\mathcal{A}_{D_{s}^{+} K^{+} \rightarrow D_{s}^{+} K^{+}}(s, t, u) .
\end{aligned}
$$

For the coupled channels with $(S, I)=(1,0)$, the strangeness-isospin amplitudes read

$$
\begin{aligned}
& \mathcal{A}_{D K \rightarrow D K}^{(1,0)}(s, t, u)=2 \mathcal{A}_{D^{0} K^{-} \rightarrow D^{0} K^{-}}(u, t, s)-\mathcal{A}_{D^{+} K^{+} \rightarrow D^{+} K^{+}}(s, t, u), \\
& \mathcal{A}_{D_{s} \eta \rightarrow D_{s} \eta}^{(1,0)}(s, t, u)=\mathcal{A}_{D_{s}^{+} \eta \rightarrow D_{s}^{+} \eta}(s, t, u), \\
& \mathcal{A}_{D_{s} \eta \rightarrow D K}^{(1,0)}(s, t, u)=-\sqrt{2} \mathcal{A}_{D_{s}^{+} K^{-} \rightarrow D^{0} \eta}(u, t, s) .
\end{aligned}
$$

For the coupled channels with $(S, I)=(1,1)$, one has

$$
\begin{aligned}
& \mathcal{A}_{D_{s} \pi \rightarrow D_{s} \pi}^{(1,1)}(s, t, u)=\mathcal{A}_{D_{s}^{+} \pi^{0} \rightarrow D_{s}^{+} \pi^{0}}(s, t, u), \\
& \mathcal{A}_{D K \rightarrow D K}^{(1,1)}(s, t, u)=\mathcal{A}_{D^{+} K^{+} \rightarrow D^{+} K^{+}}(s, t, u), \\
& \mathcal{A}_{D K \rightarrow D_{s} \pi}^{(1,1)}(s, t, u)=\sqrt{2} \mathcal{A}_{D_{s}^{+} K^{-} \rightarrow D^{0} \pi^{0}}(u, t, s) .
\end{aligned}
$$

For $(S, I)=(0,1 / 2)$, there are three channels: $D \pi, D \eta$ and $D_{s} \bar{K}$. The isospin relations are given by

$$
\begin{aligned}
\mathcal{A}_{D \pi \rightarrow D \pi}^{(0,1 / 2)}(s, t, u) & =\frac{3}{2} \mathcal{A}_{D^{+} \pi^{+} \rightarrow D^{+} \pi^{+}}(u, t, s)-\frac{1}{2} \mathcal{A}_{D^{+} \pi^{+} \rightarrow D^{+} \pi^{+}}(s, t, u), \\
\mathcal{A}_{D \eta \rightarrow D \eta}^{(0,1 / 2)}(s, t, u) & =\mathcal{A}_{D^{+} \eta \rightarrow D^{+} \eta}(s, t, u), \\
\mathcal{A}_{D_{s} \bar{K} \rightarrow D_{s} \bar{K}}^{(0,1 / 2)}(s, t, u) & =\mathcal{A}_{D_{s}^{+} K^{+} \rightarrow D_{s}^{+} K^{+}}(u, t, s), \\
\mathcal{A}_{D \eta \rightarrow D \pi}^{(0,1 / 2)}(s, t, u) & =\sqrt{3} \mathcal{A}_{D^{0} \eta \rightarrow D^{0} \pi^{0}}(s, t, u), \\
\mathcal{A}_{D_{s} \bar{K} \rightarrow D \pi}^{(0,1 / 2)}(s, t, u) & =\sqrt{3} \mathcal{A}_{D_{s}^{+} K^{-} \rightarrow D^{0} \pi^{0}}(s, t, u), \\
\mathcal{A}_{D_{s} \bar{K} \rightarrow D \eta}^{(0,1 / 2)}(s, t, u) & =\mathcal{A}_{D_{s}^{+} K^{-} \rightarrow D^{0} \eta}(s, t, u) .
\end{aligned}
$$

\subsection{Partial wave projection}

Each of the strangeness-isospin amplitudes can be denoted by $\mathcal{A}_{D_{1} \phi_{1} \rightarrow D_{2} \phi_{2}}^{(S, I)}(s, t)$. Its partial wave projection with definite angular momentum $\ell$ is given by

$$
\mathcal{A}_{\ell}^{(S, I)}(s)_{D_{1} \phi_{1} \rightarrow D_{2} \phi_{2}}=\frac{1}{2} \int_{-1}^{1} \mathrm{~d} \cos \theta P_{\ell}(\cos \theta) \mathcal{A}_{D_{1} \phi_{1} \rightarrow D_{2} \phi_{2}}^{(S, I)}(s, t(s, \cos \theta)) .
$$


Here, the Mandelstam variable $t$ is expressed in terms of $s$ and the scattering angle $\theta$,

$$
\begin{aligned}
t(s, \cos \theta)= & M_{D_{1}}^{2}+M_{D_{2}}^{2}-\frac{1}{2 s}\left(s+M_{D_{1}}^{2}-M_{\phi_{1}}^{2}\right)\left(s+M_{D_{2}}^{2}-M_{\phi_{2}}^{2}\right) \\
& -\frac{\cos \theta}{2 s} \sqrt{\lambda\left(s, M_{D_{1}}^{2}, M_{\phi_{1}}^{2}\right) \lambda\left(s, M_{D_{2}}^{2}, M_{\phi_{2}}^{2}\right)} .
\end{aligned}
$$

with $\lambda(a, b, c)=a^{2}+b^{2}+c^{2}-2 a b-2 b c-2 a c$ the Källén function. From eq. (4.18), one sees that at each of the thresholds of $D_{1} \phi_{1}$ and $D_{2} \phi_{2}$, i.e. when $s$ takes one of the following two values

$$
s_{1}=\left(M_{D_{1}}+M_{\phi_{1}}\right)^{2}, \quad s_{2}=\left(M_{D_{2}}+M_{\phi_{2}}\right)^{2},
$$

$t$ is independent of $\cos \theta$. Taking $s=s_{1}$ for instance, the $S$-wave amplitude becomes

$$
\mathcal{A}_{\ell=0}^{(S, I)}\left(s_{1}\right)_{D_{1} \phi_{1} \rightarrow D_{2} \phi_{2}}=\mathcal{A}_{D_{1} \phi_{1} \rightarrow D_{2} \phi_{2}}^{(S, I)}\left(s_{1}, t\left(s_{1}\right)\right) .
$$

This means that the $S$-wave amplitude at threshold can be obtained directly from the full amplitude by setting the energy squared at its threshold value. However, note that this simple recipe can only be used for the single channel case. For coupled channels, it is necessary to perform the partial wave projection using eq. (4.17).

Before ending this section, we remark that it is helpful to use matrix notation to denote the partial wave amplitudes with definite strangeness $S$ and isospin $I$. In the matrix notation, the subscript $D_{1} \phi_{1} \rightarrow D_{2} \phi_{2}$ is redundant. For single channels, this is apparent since the process is specified uniquely by $(S, I)$. For coupled channels, taking $(S, I)=(1,1)$ for example, there are four processes: $D_{s} \pi \rightarrow D_{s} \pi, D K \rightarrow D K, D K \rightarrow D_{s} \pi$ and its time reversal process. Using time reversal invariance, one can write

$$
\mathcal{A}_{\ell}^{(1,1)}(s)=\left(\begin{array}{ll}
\mathcal{A}_{\ell}^{(1,1)}(s)_{D_{s} \pi \rightarrow D_{s} \pi} & \mathcal{A}_{\ell}^{(1,1)}(s)_{D K \rightarrow D_{s} \pi} \\
\mathcal{A}_{\ell}^{(1,1)}(s)_{D K \rightarrow D_{s} \pi} & \mathcal{A}_{\ell}^{(1,1)}(s)_{D K \rightarrow D K}
\end{array}\right) .
$$

Later on, we will refer to the amplitudes for a given process in the isospin basis by $\mathcal{A}_{\ell}^{(S, I)}(s)_{i j}$, with $i$ and $j$ being channel indices. Unitarization of the scattering amplitudes will be discussed in the matrix notation in the following.

\subsection{Unitarization}

Unitarization is often adopted to extend ChPT to higher energies. The unitarized amplitudes sum up a series of $s$-channel loops,${ }^{6}$ which correspond to the right-hand cut, and thus one would naively expect that they can be used for higher momenta as well as larger pion masses. Phenomenologically, it is now well-known that the unitarized amplitudes can well describe the scattering data for the pion and kaon systems up to $1.2 \mathrm{GeV}$, see, e.g., refs. $[28,47]$. We thus expect that these amplitudes allows for a description of the lattice data at pion masses higher than the conventional ChPT. Yet, there is no rigorous proof a

\footnotetext{
${ }^{6}$ Since the unitarization procedure is normally equivalent to a resummation of the scattering amplitudes in the $s$-channel, it breaks the crossing symmetry. Crossing symmetry can be restored using Roy-type equations, for an early attempt, see ref. [46].
} 
priori. For varying the quark masses (or equivalently the masses of the Goldstone bosons), it provides a way to performing the chiral extrapolation of lattice simulation results or studying the quark mass dependence of physical quantities. In the present work, we will consider two different versions of unitarization for the sake of comparison and for quantifying the inherent model-dependence of such approaches. For the sake of simplicity and generality, all the quantum number indices of the amplitudes such as $S, I$ and $\ell$ will be suppressed in this section. That is to say $T, \mathcal{A}, \mathcal{T}$ and $\tilde{T}$, which will appear later on, are $T_{\ell}^{(S, I)}, \mathcal{A}_{\ell}^{(S, I)}, \mathcal{T}_{\ell}^{(S, I)}$ and $\tilde{T}_{\ell}^{(S, I)}$, respectively, for our case.

The first approach we will use is the one proposed in ref. [29], which is denoted by UChPT throughout this paper. In matrix form, the unitarized amplitude is given by

$$
T(s)=\{1-\mathcal{T}(s) \cdot g(s)\}^{-1} \cdot \mathcal{T}(s),
$$

where $g(s)$ is a diagonal matrix $g(s)=\operatorname{diag}\left\{g(s)_{i}\right\}$, with $i$ the channel index. The fundamental loop integral $g(s)_{i}$ reads

$$
g(s)_{i}=i \int \frac{\mathrm{d}^{4} q}{(2 \pi)^{4}} \frac{1}{\left(q^{2}-M_{D_{i}}^{2}+i \epsilon\right)\left((P-q)^{2}-M_{\phi_{i}}^{2}+i \epsilon\right)}, \quad s \equiv P^{2} .
$$

Note that $g(s)_{i}$ is counted as $\mathcal{O}(p)$ and its explicit expression is

$$
\begin{aligned}
g(s)_{i}=\frac{1}{16 \pi^{2}}\{ & a(\mu)+\ln \frac{M_{D_{i}}^{2}}{\mu^{2}}+\frac{s-M_{D_{i}}^{2}+M_{\phi_{i}}^{2}}{2 s} \ln \frac{M_{\phi_{i}}^{2}}{M_{D_{i}}^{2}} \\
+ & \frac{\sigma_{i}}{2 s}\left[\ln \left(s-M_{\phi_{i}}^{2}+M_{D_{i}}^{2}+\sigma_{i}\right)-\ln \left(-s+M_{\phi_{i}}^{2}-M_{D_{i}}^{2}+\sigma_{i}\right)\right. \\
& \left.\left.\quad+\ln \left(s+M_{\phi_{i}}^{2}-M_{D_{i}}^{2}+\sigma_{i}\right)-\ln \left(-s-M_{\phi_{i}}^{2}+M_{D_{i}}^{2}+\sigma_{i}\right)\right]\right\}
\end{aligned}
$$

with $\sigma_{i}=\left\{\left[s-\left(M_{\phi_{i}}+M_{D_{i}}\right)^{2}\right]\left[s-\left(M_{\phi_{i}}-M_{D_{i}}\right)^{2}\right]\right\}^{1 / 2}$ and $\mu$ the renormalization scale. One can define a $\mu$-independent parameter $\tilde{a} \equiv a(\mu)+\ln \left(M_{D_{i}}^{2} / \mu^{2}\right)$, since a change of $\mu$ in the logarithm can be compensated by $a(\mu)$. Notice that the parameter $\tilde{a}$ in $g(s)$ of eq. (4.22) cannot be absorbed by redefining the LECs. It is introduced through the dispersion integral along the right-hand cut, and is a free parameter in principle. The only constraint here is from the requirement of a proper power counting: while all other terms in eq. (4.24) are of order $\mathcal{O}(p), \tilde{a}$ should be much smaller than 1 so that its presence will not cause a breaking of the power counting if we expand the resummed amplitude to a certain order, i.e. $\tilde{a}=\mathcal{O}(p)$. The kernel matrix $\mathcal{T}(s)$ can be obtained perturbatively by matching to the ChPT amplitudes order by order. Up to NNLO, it can be expressed as

$$
\mathcal{T}(s)=\mathcal{A}^{(1)}(s)+\mathcal{A}^{(2)}(s)+\mathcal{A}^{(3)}(s)-\mathcal{A}^{(1)}(s) \cdot g(s) \cdot \mathcal{A}^{(1)}(s),
$$

where $\mathcal{A}^{(n)}(s)(n=1,2,3)$ stand for the partial wave amplitudes from the perturbative calculation with the superscript $n$ denoting the chiral dimension. Notice that the right hand cut from the NNLO amplitude is subtracted in the last term in order to avoid double counting in the unitarization. In the function $g(s)$ in the above equation, the subtraction constant $\tilde{a}$ may be removed as it can be absorbed into the redefinition of the LECs in $\mathcal{A}^{(2)}(s)$. 
The other approach is the so-called inverse amplitude method (IAM) [40-44, 47]. In our case, the IAM unitized amplitudes has the matrix form

$$
T(s)=\tilde{T}^{(1)}(s) \cdot\left[\tilde{T}^{(1)}(s)-\tilde{T}^{(2)}(s)\right]^{-1} \cdot \tilde{T}^{(1)}(s),
$$

where

$$
\tilde{T}^{(1)}(s) \equiv \mathcal{A}^{(1)}(s), \quad \tilde{T}^{(2)}(s) \equiv \mathcal{A}^{(2)}(s)+\mathcal{A}^{(3)}(s) .
$$

The above assignments guarantee that the unitarized amplitudes exactly obey unitarity when the perturbatively unitary equations are employed, i.e.,

$$
\operatorname{Im} \mathcal{A}^{(1)}(s)=0, \quad \operatorname{Im} \mathcal{A}^{(2)}(s)=0, \quad \operatorname{Im} \mathcal{A}^{(3)}(s)=\mathcal{A}^{(1)}(s) \tilde{\rho}(s) \mathcal{A}^{(1)}(s)^{\dagger},
$$

with $\tilde{\rho}(s)=\operatorname{diag}\left\{\tilde{\rho}(s)_{i}\right\}, \tilde{\rho}(s)_{i}=-q_{i} /(8 \pi \sqrt{s})$ and $q_{i}$ is the magnitude of the center-of-mass (CM) three-momentum in the $i^{\text {th }}$ channel.

\section{Calculation of the scattering lengths}

\subsection{Definition and pion mass dependence}

Given definite strangeness $S$ and isospin $I$, the $S$-wave scattering lengths of the $i^{\text {th }}$ channel are related to the diagonal elements of the $T$-matrix, ${ }^{7}$

$$
a_{i}^{(S, I)}=-\frac{1}{8 \pi\left(M_{D_{i}}+M_{\phi_{i}}\right)} T_{\ell=0}^{(S, I)}\left(s_{\mathrm{th}}\right)_{i i}, \quad s_{\mathrm{th}}=\left(M_{D_{i}}+M_{\phi_{i}}\right)^{2} .
$$

Here, $M_{D_{i}}$ and $M_{\phi_{i}}$ denote the masses of the charmed meson and Goldstone boson $\phi$ in the channel $i$, respectively, and $T_{\ell=0}^{(S, I)}\left(s_{\mathrm{th}}\right)$ stands for the $S$-wave unitarized amplitude at threshold using either UChPT given by eq. (4.22) or IAM given by eq. (4.26).

Due to the short lifetime of the charmed meson, there are no experimental data for $D-\phi$ scattering lengths. Nevertheless, lattice QCD calculations in the last a few years provide very valuable information on the interaction between the charmed mesons and light pseudoscalar mesons [15-18]. Since the lattice calculations were performed at several unphysical pion masses, in order to describe these lattice data, one should know the pion mass dependence of the scattering lengths. This is achieved by replacing all the quantities in the expressions by the pion mass dependent ones. For the involved meson masses, we have

$$
M_{K}=\sqrt{\stackrel{\stackrel{\circ}{M}_{K}^{2}+M_{\pi}^{2} / 2}{2}}, \quad M_{D}=\stackrel{\circ}{M}_{D}+\left(h_{1}+2 h_{0}\right) \frac{M_{\pi}^{2}}{\stackrel{\circ}{M}_{D}}, \quad M_{D_{s}}=\stackrel{\circ}{M_{D_{s}}}+2 h_{0} \frac{M_{\pi}^{2}}{\stackrel{\circ}{M}_{D_{s}}} .
$$

Note that all the formulae shown above are of NLO for the pion mass dependence. ${ }^{8}$ Here the LEC $h_{1}$ can be fixed by the mass difference between $D$ and $D_{s}$. Using these two

\footnotetext{
${ }^{7}$ We are using the sign convention such that the scattering length for a repulsive interaction is negative.

${ }^{8} \mathrm{In}$ eq. (5.2), although the formula we used for the kaon mass is a LO expression in $\mathrm{SU}(3) \mathrm{ChPT}$, it contains two parts: the part $\sim \stackrel{\circ}{M}_{K}^{2}$ proportional to $B_{0} m_{s}$ remains in the $\mathrm{SU}(2)$ chiral limit and is regarded as a LO contribution of the pion mass dependence, while the part related to $B_{0} m_{u / d} \sim M_{\pi}^{2} / 2$ vanishes in the $\mathrm{SU}(2)$ chiral limit and is thus a NLO contribution. In this sense, we spelled out the pion mass dependence for all of the masses and decay constants consistently up to the order $M_{\pi}^{2}$.
} 
equations, one has [12]

$$
h_{1}=\frac{M_{D_{s}}^{2}-M_{D}^{2}}{4\left(M_{K}^{2}-M_{\pi}^{2}\right)}=0.4266
$$

where the physical values for the meson masses are used, i.e., $M_{\pi}=138 \mathrm{MeV}$, $M_{K}=496 \mathrm{MeV}, M_{D}=1867 \mathrm{MeV}$ and $M_{D_{s}}=1968 \mathrm{MeV} \cdot{ }^{9}$ The pion decay constant should also be substituted by [32]

$$
F_{\pi}=F_{0}\left\{1-2 \mu_{\pi}-\mu_{K}+\frac{4 M_{\pi}^{2}}{F_{0}^{2}}\left[L_{4}^{r}(\mu)+L_{5}^{r}(\mu)\right]+\frac{8 M_{K}^{2}}{F_{0}^{2}} L_{4}^{r}(\mu)\right\},
$$

where $M_{K}$ is understood as the one in eq. (5.2), and $\mu_{\phi}$ is a scale dependent function for the Goldstone boson $\phi$

$$
\mu_{\phi}=\frac{M_{\phi}^{2}}{32 \pi^{2} F_{0}^{2}} \ln \frac{M_{\phi}^{2}}{\mu^{2}} .
$$

So far, except for $h_{1}$, the LECs $L_{4}^{r}, L_{5}^{r}$ and $h_{0}$ and the chiral limit quantities $\stackrel{\circ}{K}_{K}, \stackrel{\circ}{M}_{D}$, $\stackrel{\circ}{M}_{D_{s}}$ and $F_{0}$ are all unknown. Since we will fit to the lattice results on the scattering lengths calculated in ref. [16], we choose to fix the above mentioned quantities from fitting to the lattice data calculated using the same gauge configurations. In addition, the kaon decay constant $F_{K}$ data are also included to have a bigger data set for fixing $F_{0}$ and $L_{4,5}^{r}$. The pion mass dependence of $F_{K}$ is given by [32]

$$
F_{K}=F_{0}\left\{1-\frac{3}{4}\left(\mu_{\pi}+2 \mu_{K}+\mu_{\eta}\right)+\frac{4 M_{\pi}^{2}}{F_{0}^{2}} L_{4}^{r}(\mu)+\frac{4 M_{K}^{2}}{F_{0}^{2}}\left[2 L_{4}^{r}(\mu)+L_{5}^{r}(\mu)\right]\right\},
$$

with $M_{\eta}^{2}=\left(4 M_{K}^{2}-M_{\pi}^{2}\right) / 3$ and $M_{K}$ given by eq. (5.2).

The lattice data for $M_{K}, M_{D}$ and $M_{D_{s}}$ are taken from ref. [16]. There are four data sets for each quantity, corresponding to the four ensembles (labelled by M007, M010, M020 and M030) with pion mass approximately $301.1 \mathrm{MeV}, 363.8 \mathrm{MeV}, 511.0 \mathrm{MeV}$ and $617.0 \mathrm{MeV}$, in order. Since the same ensembles are employed in ref. [48], we take the data for $f_{\pi}$ and $f_{K}$ from ref. [48], where $f_{\pi}=\sqrt{2} F_{\pi}$ and $f_{K}=\sqrt{2} F_{K}$. Those lattice data are well described as shown in figure $3^{10}$ when the parameters take the values given in table 3. Our fitting values for $L_{4,5}^{r}$ are consistent with the determinations given in refs. $[49,50]$. Therein, the values are obtained at $\mu=M_{\eta}$, and the corresponding values transformed to $\mu=M_{\rho}$ can be found in ref. [51].

\subsection{Fits to lattice data on the scattering lengths}

\subsubsection{Introduction to the fitting procedure}

Since all the necessary preparations are completed, we proceed to the description of the lattice QCD data of the $S$-wave scattering lengths. There are two points to be discussed before carrying out the fits.

\footnotetext{
${ }^{9}$ The mass of the $\eta$ is always expressed in terms of $M_{\pi}$ and $M_{K}$ through the Gell-Mann-Okubo mass relation, $3 M_{\eta}^{2}=4 M_{K}^{2}-M_{\pi}^{2}$.

${ }^{10}$ We have neglected the subtleties due to the use of mixed action gauge configurations in the lattice calculations, which in principle requires to use the partially quenched ChPT instead of the standard one for the chiral extrapolation, and the effect of finite lattice spacing, see ref. [48].
} 

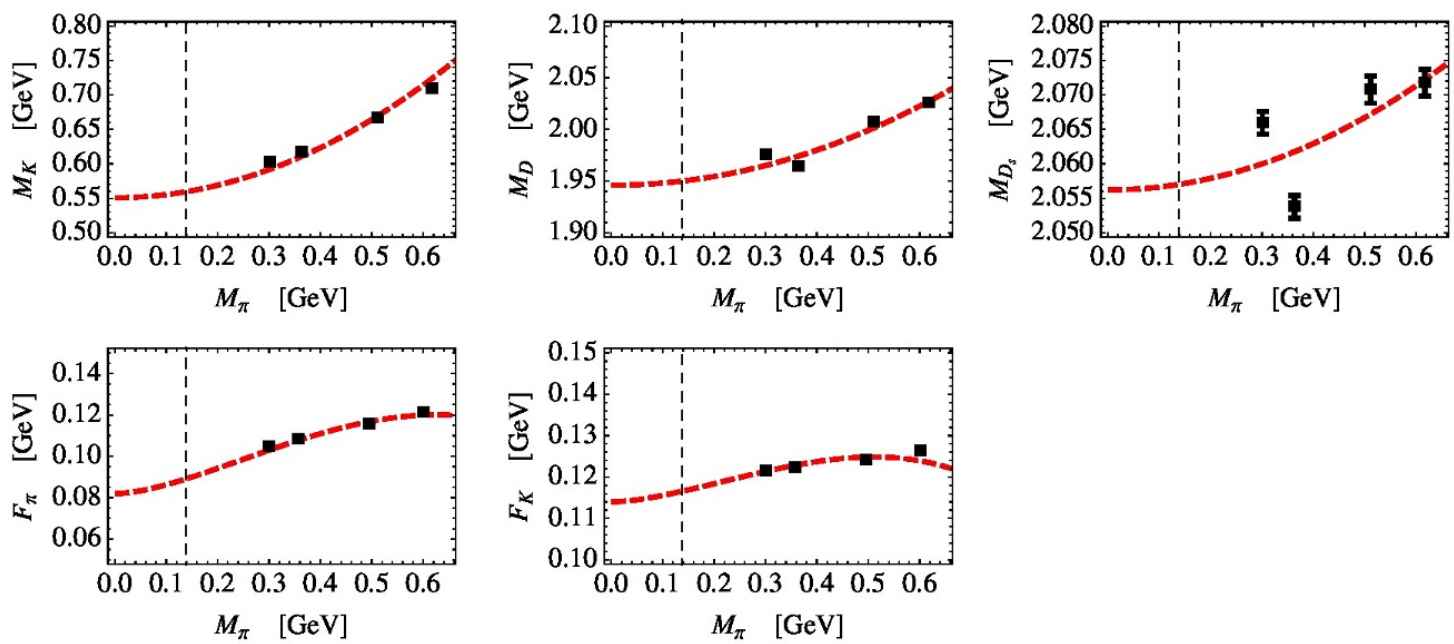

Figure 3. Chiral extrapolation of masses and decay constants. All the lattice data are obtained from the same ensembles, namely M007-M030. Data for $M_{K}, M_{D}$ and $M_{D_{s}}$ is taken from ref. [16] and the one for $F_{\pi}$ and $F_{K}$ from ref. [48]. Except for $M_{D_{s}}$, the data errors are so tiny that we do not show them explicitly in the plots. The vertical dashed line corresponds to the physical pion mass.

\begin{tabular}{|cccccccc|}
\hline$\stackrel{\circ}{M}_{K}$ & $\stackrel{\circ}{M}_{D}$ & $\stackrel{\circ}{M}_{D_{s}}$ & $h_{0}$ & $h_{1}$ & $F_{0}$ & $10^{3} \cdot L_{4}^{r}$ & $10^{3} \cdot L_{5}^{r}$ \\
\hline 560.41 & 1940.4 & 2061.2 & 0.0172 & $0.4266^{*}$ & 73.31 & 0.0095 & 1.3264 \\
\hline
\end{tabular}

Table 3. Parameters for chiral extrapolation. $L_{4}^{r}$ and $L_{5}^{r}$ are obtained at $\mu=M_{\rho}(=775.5 \mathrm{MeV})$. The masses and decay constant in the chiral limit are in units of MeV. $h_{0}$ and $h_{1}$ and dimensionless. The asterisk marks an input value.

The first one is related to the lattice data. From ref. [16], 20 data for 5 channels are available. Amongst the five channels, the $D_{s} \pi$ with $(S, I)=(1,1)$ can actually be coupled to the isovector $D K$ channel while the other four are single channels. Although in ref. [16] only the $D_{s} \pi$ interpolating operator was constructed and used, the propagation of all the quarks should know about the presence of the coupled $D K$ channel with $(S, I)=(1,1)$ because the channel-coupling in this case does not require disconnected Wick contractions which were not included in ref. [16]. Thus, we will describe the $D_{s} \pi$ data using a coupled-channel unitarized amplitude.

In addition, lattice QCD results were published in the last two years for two more channels: $D \pi$ with $(S, I)=(0,1 / 2)[17]$ and $D K$ with $(S, I)=(1,0)$ [18]. These channels are more difficult since both of them involve disconnected Wick contractions, ${ }^{11}$ but they are also more interesting as they can provide valuable information for the lightest scalar charmed mesons in the corresponding channels. The calculation for the $D \pi$ scattering was

\footnotetext{
${ }^{11}$ It is shown in ref. [52] that as long as the singly disconnected Wick contractions contribute, which is the case for the isoscalar $D K$ channel, they are of LO in both the $1 / N_{c}$ and chiral expansion. Therefore, they cannot be neglected.
} 
performed using $N_{f}=2$ gauge configurations, and the $D K$ calculation has results from both $N_{f}=2$ and $N_{f}=2+1$ gauge configurations. Because the amplitudes derived here are based on $\mathrm{SU}(3) \mathrm{ChPT}$, we will only include in the fits the new result with $N_{f}=2+1$, i.e. $a_{D K \rightarrow D K}^{(1,0)}=-1.33(20) \mathrm{fm}$ obtained at $M_{\pi}=156 \mathrm{MeV}$, and the unitarized amplitude used in the fits is obtained including the $D_{s} \eta$ coupled channel. Notice that these new lattice calculations use gauge configurations and actions different from those in ref. [16], the chiral-limit masses for the kaon and charmed mesons should take different values from those given in table 3. Because the physical masses of the involved ground state mesons such as the kaon and charmed mesons were reproduced rather well with the lattice setup used in ref. [18] (for details, see ref. [19]), the chiral-limit values of the involved meson masses and $F_{0}$ are determined by requiring them to coincide with the corresponding phyiscal values at the physical pion mass, namely, $\stackrel{\circ}{M}_{K}=486.3 \mathrm{MeV}, \stackrel{\circ}{M}_{D}=1862.3 \mathrm{MeV}, \stackrel{\circ}{M}_{D_{s}}=1967.7 \mathrm{MeV}$ and $F_{0}=76.23 \mathrm{MeV}$. The values for the LECs in the extrapolating expressions of these quantities are the same as those listed in table 3.

The other point concerns the LECs to be determined. There are 7 unknown LECs in total: $h_{2}, h_{3}, h_{4}, h_{5}, g_{1}, g_{2}$ and $g_{3}$. As mentioned in the previous work [16], $h_{2}\left(h_{3}\right)$ and $h_{4}\left(h_{5}\right)$ are largely correlated. Therefore, redefinitions of the LECs are employed to reduce these correlations, which are

$$
h_{24}=h_{2}+h_{4}^{\prime}, \quad h_{35}=h_{3}+2 h_{5}^{\prime}, \quad h_{4}^{\prime}=h_{4} \bar{M}_{D}^{2}, \quad h_{5}^{\prime}=h_{5} \bar{M}_{D}^{2} .
$$

The new parameters $h_{24}, h_{35}, h_{4}^{\prime}$ and $h_{5}^{\prime}$ will be determined in our fits. The average of the physical masses of the charmed $D$ and $D_{s}$ mesons, $\bar{M}_{D}=\left(M_{D}^{\text {phy }}+M_{D_{s}}^{\text {phy }}\right) / 2$, is introduced to make the four new parameters dimensionless. Similarly, for the LECs from the NNLO contact terms, $g_{2}$ and $g_{3}$ are largely correlated with each other, and it is better to redefine these LECs as

$$
g_{23}=g_{2}^{\prime}-2 g_{3}^{\prime}, \quad g_{1}^{\prime}=g_{1} \bar{M}_{D}, \quad g_{2}^{\prime}=g_{2} \bar{M}_{D}, \quad g_{3}^{\prime}=g_{3} \bar{M}_{D}^{3} .
$$

The parameters $g_{1}^{\prime}, g_{23}$ and $g_{3}^{\prime}$ have a dimension of inverse mass and will be fixed from fitting to the lattice data. One can fix $g_{1}^{\prime}$ and $g_{23}$ separately only when the coupled-channel unitarized amplitudes are used, i.e. from fitting to the lattice results of the $D_{s} \pi$ and the isoscalar $D K$ scattering lengths. The single-channel unitarized amplitudes is only sensitive to the combination $g_{123}=g_{23}-g_{1}^{\prime}$, instead of $g_{1}^{\prime}$ and $g_{23}$ separately, and $g_{3}^{\prime}$.

\subsubsection{Results}

We will try different fit procedures. In the fit UChPT-6(a), all of the 20 data points for 5 channels, with pion masses from $301 \mathrm{MeV}$ up to $617 \mathrm{MeV}$, in ref. [16] as well as the $N_{f}=2+1$ datum for the isoscalar $D K$ channel, with an almost physical pion mass of $156 \mathrm{MeV}$, in ref. [18] are taken into consideration. We notice that there are two possibilities for a scattering length to be negative in our sign convention: a repulsive interaction, and an attractive interaction with a bound state pole below the threshold. In the $(S, I)=(1,0)$ channel, there is the well-known state $D_{s 0}^{*}(2317)$ below the $D K$ threshold which was not included as an explicit degree of freedom in our theory. Because the number of data is 


\begin{tabular}{|rrrrr|}
\hline & $\begin{array}{rrr}\text { UChPT-6(a) } \\
\text { no prior }\end{array}$ & no prior & with prior & with prior \\
\hline$h_{24}$ & $0.79_{-0.09}^{+0.10}$ & $0.76_{-0.09}^{+0.10}$ & $0.83_{-0.10}^{+0.11}$ & $0.80_{-0.10}^{+0.10}$ \\
$h_{35}$ & $0.73_{-0.38}^{+0.50}$ & $0.81_{-0.62}^{+0.95}$ & $0.43_{-0.23}^{+0.23}$ & $0.40_{-0.29}^{+0.33}$ \\
$h_{4}^{\prime}$ & $-1.49_{-0.57}^{+0.55}$ & $-1.56_{-0.65}^{+0.61}$ & $-1.33_{-0.60}^{+0.60}$ & $-1.72_{-0.63}^{+0.64}$ \\
$h_{5}^{\prime}$ & $-11.47_{-2.79}^{+2.24}$ & $-15.38_{-7.20}^{+4.81}$ & $-4.25_{-0.66}^{+0.65}$ & $-2.60_{-0.87}^{+0.84}$ \\
$g_{1}^{\prime}$ & $-1.66_{-1.59}^{+0.31}$ & $-2.44_{-0.64}^{+0.57}$ & $-1.10_{-0.23}^{+0.18}$ & $-1.90_{-0.35}^{+0.58}$ \\
$g_{23}$ & $-1.24_{-1.51}^{+0.28}$ & $-2.00_{-0.51}^{+0.52}$ & $-0.70_{-0.24}^{+0.19}$ & $-1.48_{-0.37}^{+0.61}$ \\
$g_{3}^{\prime}$ & $2.12_{-0.45}^{+0.55}$ & $2.85_{-0.96}^{+1.41}$ & $0.98_{-0.14}^{+0.15}$ & $0.58_{-0.19}^{+0.20}$ \\
\hline$\chi^{2} /$ d.o.f. & $\frac{31.52}{21-7}=2.25$ & $\frac{13.43}{16-7}=1.49$ & $\frac{77.72-23.34}{21-7}=3.88$ & $\frac{51.71-16.60}{16-7}=3.90$ \\
\hline
\end{tabular}

Table 4. Values of the LECs from the 6-channel fits using the method of UChPT. The $h_{i}$ 's are dimensionless, and the $g_{1}^{\prime}, g_{23}$ and $g_{3}^{\prime}$ are in $\mathrm{GeV}^{-1}$.

small but the number of parameters is large, a direct fit to these lattice data might result in solutions which are not physically acceptable. For instance, within the range of the parameters of a direct fit, the $(S, I)=(1,0) D K$ channel could even be repulsive which is reflected by the fact that the kernel of the unitarized amplitude takes a positive value at the threshold. Given that the LO interaction in the corresponding $D K$ channel is the most attractive one among all the charmed meson-Goldstone boson scattering processes, see table II in ref. [16] for instance, we regard such a situation as unacceptable. Therefore, we put a constraint by hand requiring that when all the particles take their physical values there is a bound state pole in the $(S, I)=(1,0)$ channel at $2317 \mathrm{MeV}$. Following ref. [16], this is done by adjusting the subtraction constant $\tilde{a}$ in the loop function $g(s)$ in the unitarized amplitude, eq. (4.22), to produce the pole at the right position. The resulting values of the LECs from the fit are shown in table 4 .

However, a pion mass larger than $600 \mathrm{MeV}$ is definitely too large for the chiral extrapolation using the standard ChPT. The unitarized approach arguably has a larger convergence range than the standard ChPT. But the range is not known a priori. Therefore, for the sake of comparison, we perform another fit, denoted as UChPT-6(b), using the same method but excluding the lattice data at $M_{\pi}=617 \mathrm{MeV}$. The fit results are shown in the third column of table 4. One can see that the values of all the LECs from these two fits are similar, but those from UChPT-6(b) have larger uncertainties as a result of being less constrained. The fit results from both fits are plotted in figure 4 . The bands represent the variation of the scattering lengths with respect to the LECs within 1- $\sigma$ standard deviation. As we can see, both fits describe the lattice data reasonably well with the exception that the isoscalar $D K$ 

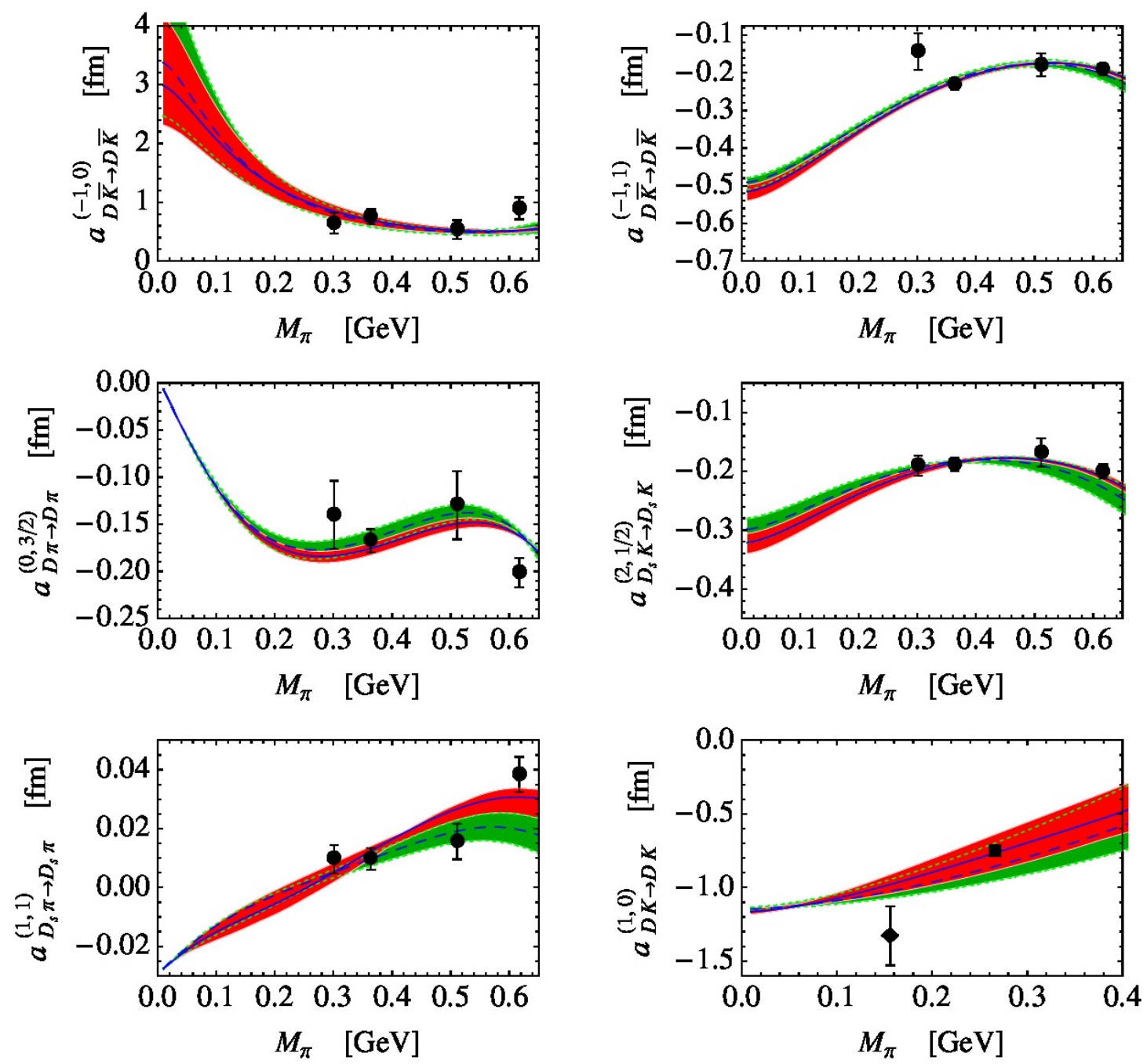

Figure 4. Comparison of the results of the 6-channel fits (without a prior $\chi^{2}$ ) to the lattice data of the scattering lengths. U $\chi$ PT-6(a): solid blue line with red band, U $\chi$ PT- $6(\mathrm{~b})$ : dashed blue line with green band. The filled circles are lattice results in ref. [16], and the filled square (not included in the fits) and diamond are taken from ref. [18].

scattering length around $M_{\pi}=156 \mathrm{MeV}$ is too large in comparison with the lattice result. However, both fits are consistent with the $N_{f}=2$ lattice result for $D K$ at a pion mass around $266 \mathrm{MeV}$ which was not included in the fits. We notice that the lattice ensemble for the $M_{\pi}=156 \mathrm{MeV}$ datum has a rather small volume with $M_{\pi} L \approx 2.3$. It is a bit too small for Lüscher's finite volume formalism to be strictly applicable, and thus this datum might bear a large systematic uncertainty. The isospin-3/2 $D \pi \rightarrow D \pi$ scattering length vanishes at the chiral limit as required by chiral symmetry. Lattice discretization often breaks chiral symmetry. However, due to the use of the domain-wall action for the valence quarks in the lattice calculation of the pionic channels, the chiral behavior is protected in our case. For related discussions in mixed-action ChPT, we refer to refs. [53-56]. 

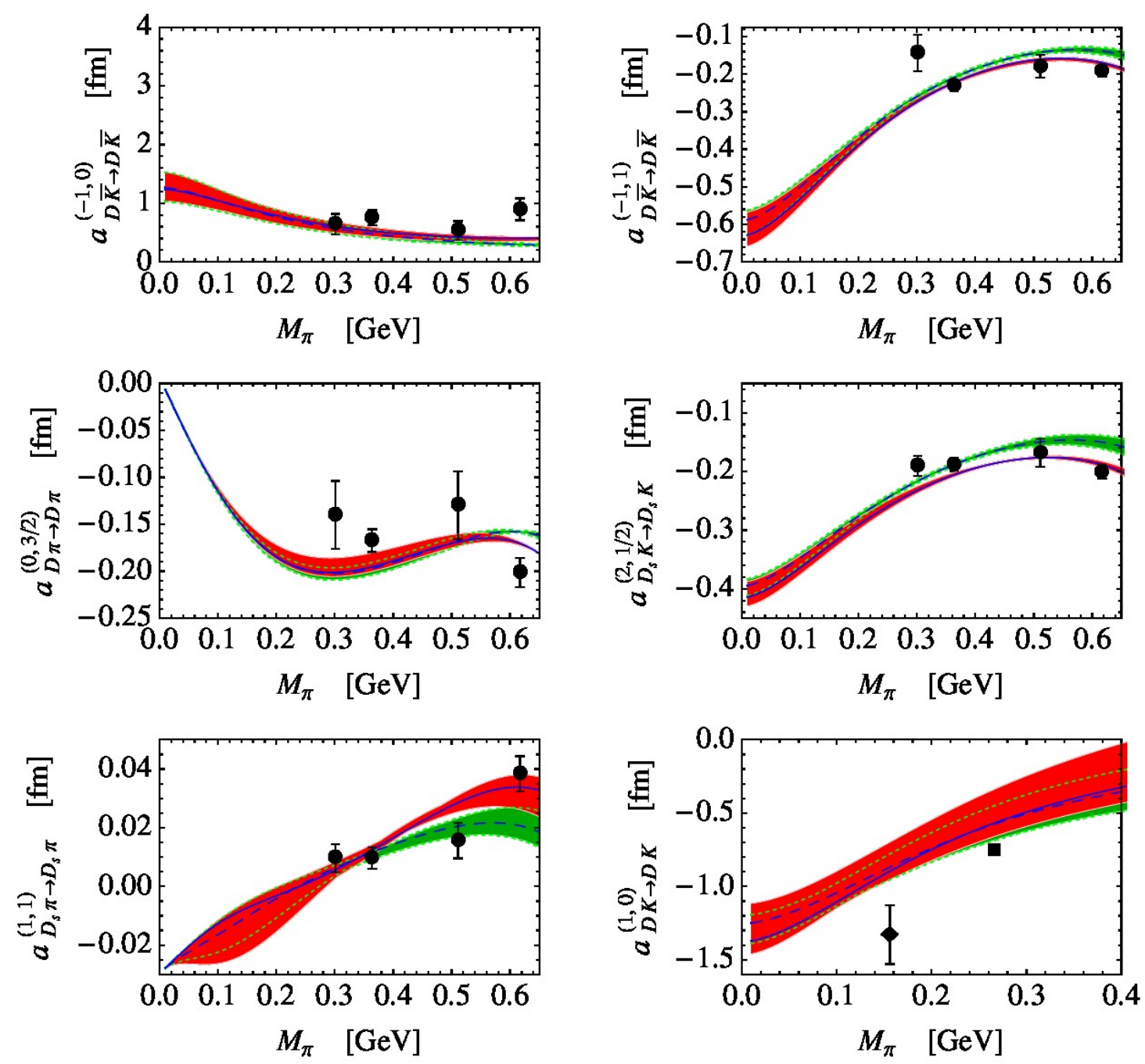

Figure 5. Comparison of the results of the 6-channel fits (with a prior $\chi^{2}$ ) to the lattice data of the scattering lengths. U $\chi$ PT-6(a): solid blue line with red band, U $\chi$ PT-6(b): dashed blue line with green band. The filled circles are lattice results in ref. [16], and the filled square (not included in the fits) and diamond are taken from ref. [18].

In both fits, the values of all the LECs except for $h_{5}^{\prime}$ turn out to be of a natural size. However, the absolute value of the dimensionless LEC $h_{5}^{\prime}$ is too large to be natural. This means that the absolute value of $h_{5}^{\prime}$ is so large that this single term would give a contribution larger than the LO amplitude. It would spoil the convergence, and thus the perturbative expansion, at least for some quantities (although for some other quantities, due to fine-tuned cancellation the sum of the NLO contribution could still be much smaller than the LO one). Therefore, we try to constrain all the LECs to natural values following ref. [57] which discusses the use of the Bayesian method in effective field theories. Following 


\begin{tabular}{|crr|}
\hline & UChPT-4 & IAM-4 \\
\hline$h_{24}$ & $0.50_{-0.10}^{+0.09}$ & $0.53_{-0.07}^{+0.07}$ \\
$h_{35}$ & $-0.89_{-0.91}^{+0.93}$ & $-0.59_{-1.11}^{+1.04}$ \\
$h_{4}^{\prime}$ & $1.23_{-1.08}^{+1.03}$ & $0.64_{-0.66}^{+0.66}$ \\
$h_{5}^{\prime}$ & $-3.09_{-4.72}^{+4.69}$ & $-6.08_{-5.99}^{+6.05}$ \\
$g_{123}$ & $0.18_{-0.18}^{+0.18}$ & $0.23_{-0.22}^{+0.21}$ \\
$g_{3}^{\prime}$ & $1.01_{-0.86}^{+0.87}$ & $1.42_{-1.10}^{+1.08}$ \\
\hline$\chi^{2} /$ d.o.f. & $\frac{13.59}{16-6}=1.36$ & $\frac{13.97}{16-6}=1.40$ \\
\hline
\end{tabular}

Table 5. Values of the LECs from the 4-channel fits using both the methods of UChPT and IAM. The $h_{i}$ 's are dimensionless, and the $g_{123}=g_{23}-g^{\prime}$ and $g_{3}^{\prime}$ are in $\mathrm{GeV}^{-1}$.

that paper, the so-called augmented chi-squared can be defined by ${ }^{12}$

$$
\chi_{\text {aug }}^{2}=\chi^{2}+\chi_{\text {prior }}^{2},
$$

where $\chi^{2}$ is the usual chi-squared used in the standard least chi-squared fit and $\chi_{\text {prior }}^{2}$ is a prior chi-squared encoding the naturalness requirement of the fit parameters. In our specific case, the $\chi_{\text {prior }}^{2}$ is set to be the sum of squares of the fit LECs. This means that we require the dimensionless LECs $h_{i}^{(\prime)}$ 's to be $\mathcal{O}(1)$ and $g_{i}^{\prime}$ 's to be $\mathcal{O}\left(1 \mathrm{GeV}^{-1}\right)$. The results by minimizing the augmented chi-squared are listed in the last two columns in table 4, denoted as UChPT-6 $\left(a^{\prime}\right)$ and UChPT-6 $\left(b^{\prime}\right)$, where the values for $\chi^{2}$ are given with $\chi_{\text {prior }}^{2}$ subtracted. One sees that the value of $h_{5}^{\prime}$ gets more natural at the price of a larger $\chi^{2}$. A comparison of the scattering lengths with the lattice data in various channels is given in figure 5 , and one can see that the lattice data in all six channels can still be described reasonably well.

It turns out that in all of these fits $\left|h_{5}^{\prime}\right|>\left|h_{4}^{\prime}\right|$, which is consistent with the $N_{c}$ counting $\left|h_{4}^{\prime}\right|=\mathcal{O}\left(\left|h_{5}^{\prime}\right| / N_{c}\right)$ [16]. The values of the $h_{i}$ 's are different from those obtained in ref. [16]. The reason may be attributed to the use of the EOMS scheme in this work, and all of $h_{2,3,4,5}$ absorb a power counting breaking contribution, see eq. (3.11). For the case of the $D_{s} \pi$, the scattering length does not vanish at the limit of a vanishing pion mass. This is due to the presence of the $D K$-loop in the coupled-channel amplitude which has a nonvanishing contribution in the $\mathrm{SU}(2)$ chiral limit. We have checked that the elastic contribution tends to zero as $M_{\pi}$ approaches zero as required by chiral symmetry.

For comparison, we also perform fits with just the four single-channel data, i.e. the $D_{s} \pi$ and isoscalar $D K$ data are excluded. For this case, we use two different unitarization

\footnotetext{
${ }^{12}$ The method in ref. [57] was only derived for the case that the dependence on the parameters to be fitted is linear. Although our case is non-linear and thus the augmented $\chi^{2}$ lacks a strict statistical meaning, we still try this method as the $\chi^{2}$ defined in this way comprises a "naturalness prior" so as to favor natural values for the LECs.
} 

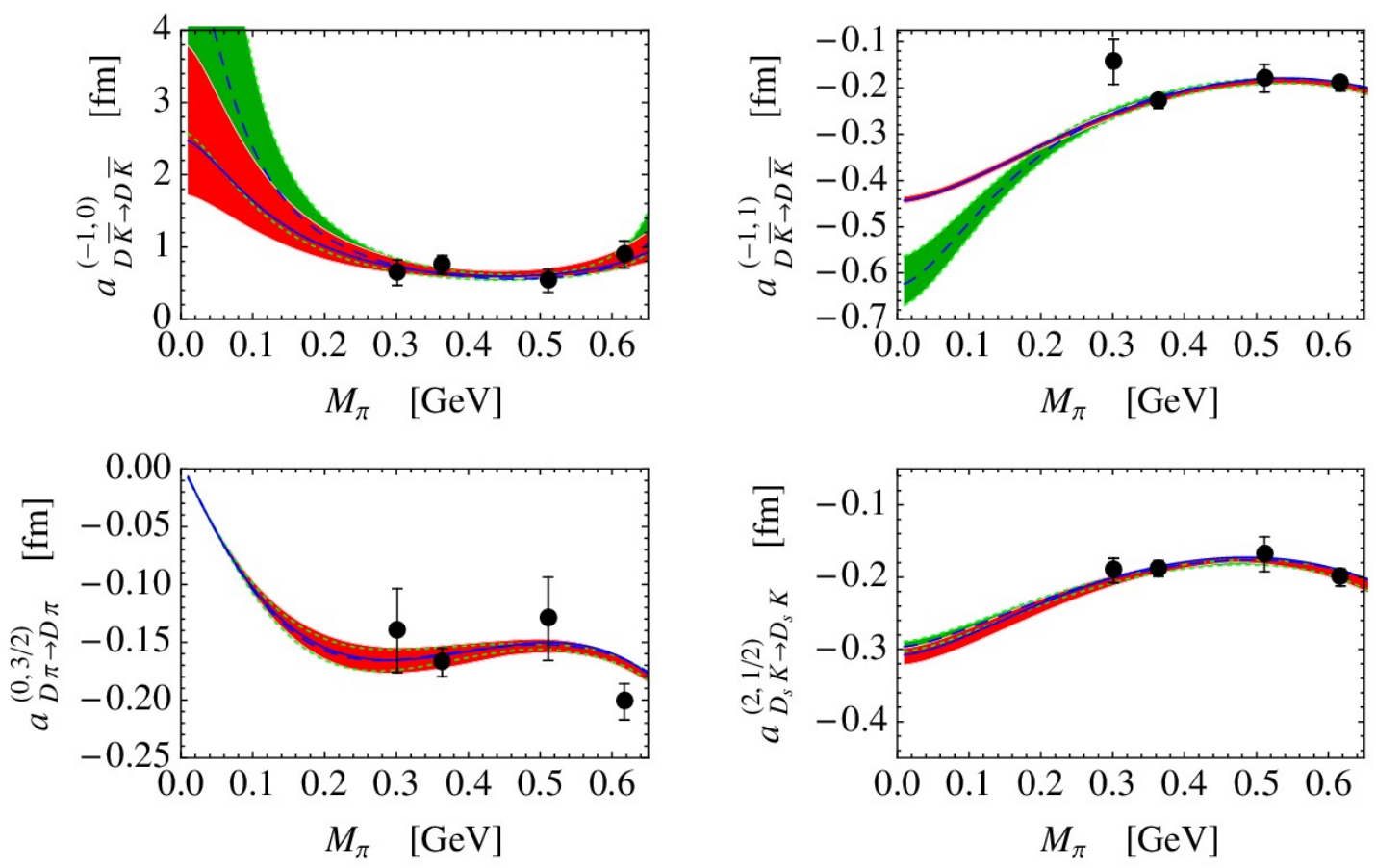

Figure 6. Comparison of the results of the 4-channel fits to the lattice data of the scattering lengths. U $\chi \mathrm{PT}-4$ : solid red line with blue band, IAM-4: dashed red line with green band. The lattice data are taken from ref. [16].

methods: UChPT, to be denoted as UChPT-4, and IAM, to be denoted as IAM-4. We did not use the IAM approach in the 6-channel fits because this approach is not suitable to unitarize a perturbative amplitude with a zero LO contribution. As can be seen from eq. (4.26), if the LO amplitude vanishes the unitarized one will vanish as well. This happens to the case of the $D_{s} \pi$. The UChPT approach is free of this problem. The results of these two fits are compiled in table 5. Notice that in this case $g_{1}^{\prime}$ and $g_{23}$ cannot be determined separately, and the effective combined parameter is $g_{123}=g_{23}-g_{1}^{\prime}$. One sees that the values of LECs from the fits using different unitarization methods are consistent with each other, ${ }^{13}$ but are only marginally consistent with those in the 6 -channel fits. In addition, the uncertainties are quite large. More lattice simulations are apparently necessary to pin down the LEC values. A comparison of the results of the 4-channel fits to the lattice data in these channels are plotted in figure 6 .

For reference, the values for the scattering lengths extrapolated to the physical pion mass are presented in table 6 . The chiral limit values in table 3 are adopted for all the 16 channels when performing the chiral extrapolation. Here we only show the results using the 6-channel fits to the data with the pion mass up to $511 \mathrm{MeV}$, i.e. UChPT-6(b) and

\footnotetext{
${ }^{13}$ However, not all of the LECs in these different unitarization methods ought to take the same values. One can see this by expanding the IAM resummed amplitude up to $\mathcal{O}\left(p^{3}\right)$. Considering the single channel case for simplicity, one has $T_{\mathrm{IAM}}(s)=\mathcal{A}^{(1)}(s)+\mathcal{A}^{(2)}(s)+\mathcal{A}^{(3)}(s)+\left[\mathcal{A}^{(2)}(s)\right]^{2} / \mathcal{A}^{(1)}(s)+\mathcal{O}\left(p^{4}\right)$. It is different from that of $\operatorname{UChPT}, T_{\mathrm{UChPT}}(s)=\mathcal{A}^{(1)}(s)+\mathcal{A}^{(2)}(s)+\mathcal{A}^{(3)}(s)+\mathcal{O}\left(p^{4}\right)$. Thus, the LECs in the $\mathcal{O}\left(p^{3}\right)$ Lagrangian could take different values.
} 


\begin{tabular}{|lcc|}
\hline$a_{i i}^{(S, I)}$ & $\mathrm{UChPT}-6(\mathrm{~b})$ & $\mathrm{UChPT}-6\left(b^{\prime}\right)$ \\
\hline$a_{D \bar{K} \rightarrow D \bar{K}}^{(-1,0)}$ & $1.76_{-0.31}^{+0.39}$ & $0.93_{-0.15}^{+0.15}$ \\
$a_{D \bar{K} \rightarrow D \bar{K}}^{(-1,1)}$ & $-0.40_{-0.01}^{+0.01}$ & $-0.45_{-0.02}^{+0.01}$ \\
$a_{D \pi \rightarrow D \pi}^{\left(0, \frac{1}{2}\right)}$ & $0.65_{-0.09}^{+0.11}$ & $0.42_{-0.05}^{+0.04}$ \\
$a_{D \eta \rightarrow D \eta}^{\left(0, \frac{1}{2}\right)}$ & $-0.18_{-0.04}^{+0.04}+i 0.00_{-0.00}^{+0.01}$ & $-0.21_{-0.04}^{+0.05}+i 0.01_{-0.01}^{+0.01}$ \\
$a_{D_{s} \bar{K} \rightarrow D_{s} \bar{K}}^{\left(0, \frac{1}{2}\right)}$ & $-1.37_{-0.04}^{+0.21}+i 0.61_{-0.02}^{+0.45}$ & $-0.47_{-0.07}^{+0.06}+i 0.50_{-0.16}^{+0.18}$ \\
$a_{D \pi \rightarrow D \pi}^{\left(0, \frac{3}{2}\right)}$ & $-0.14_{-0.01}^{+0.01}$ & $-0.15_{-0.01}^{+0.01}$ \\
$a_{D K \rightarrow D K}^{(1,0)}$ & $-1.04_{-0.03}^{+0.06}$ & $-1.50_{-0.26}^{+0.13}$ \\
$a_{D_{s} \eta \rightarrow D_{s} \eta}^{(1,0)}$ & $-0.62_{-0.03}^{+0.02}+i 0.01_{-0.00}^{+0.01}$ & $-0.76_{-0.05}^{+0.05}+i 0.05_{-0.01}^{+0.00}$ \\
$a_{D_{s} \pi \rightarrow D_{s} \pi}^{(1,1)}$ & $-0.01_{-0.01}^{+0.01}$ & $-0.01_{-0.01}^{+0.01}$ \\
$a_{D K \rightarrow D K}^{(1,1)}$ & $-1.11_{-0.09}^{+0.23}+i 0.77_{-0.04}^{+0.27}$ & $-0.82_{-0.38}^{+0.59}+i 1.64_{-0.11}^{+0.01}$ \\
$a_{D_{s} K \rightarrow D_{s} K}^{\left(2, \frac{1}{2}\right)}$ & $-0.25_{-0.02}^{+0.01}$ & $-0.32_{-0.01}^{+0.01}$ \\
\hline
\end{tabular}

Table 6. Predictions of the scattering lengths at physical pion mass using the LECs determined in the 6-channel fits UChPT-6(b) and UChPT-6( $\left.b^{\prime}\right)$ in units of fm.

UChPT-6 $\left(b^{\prime}\right)$. We notice that the numerical results of the scattering lengths extrapolated to the physical pion masses in some channels differ from those obtained in ref. [16]. This could indicate that the uncertainties are underestimated as the SU(3) formalism for UChPT was applied to pion masses higher than $500 \mathrm{MeV}$. We expect that the situation will improve when lattice results at lower pion masses are availble.

\subsubsection{Contribution of vector charmed mesons}

In this section, contributions from vector charmed mesons will be included explicitly in order to quantify their influences on the $S$-wave scattering lengths. The diagrams that survive in the heavy quark limit, see also ref. [24], are taken into account and shown in figure 7. Those diagrams vanishing in the heavy quark limit are suppressed by $1 / m_{c}$ and therefore are neglected. We denote the vector charmed mesons by $D^{*}=\left(D^{* 0}, D^{*+}, D_{s}^{*+}\right)$, and the vertices involved in figure 7 are described by the following Lagrangian,

$$
\mathcal{L}_{D^{*} D \Phi}=-\mathcal{D}_{\mu} D^{* \nu} \mathcal{D}^{\mu} D_{\nu}^{* \dagger}+M_{0}^{*} D^{* \nu} D_{\nu}^{* \dagger}+i \tilde{g}\left(D_{\mu}^{*} u^{\mu} D^{\dagger}-D u^{\mu} D_{\mu}^{* \dagger}\right)
$$

where the covariant derivatives acting on $D^{*}$ are analogous to those defined in eq. (2.10). Further, $M_{0}^{*}$ is the mass of $D^{*}$ in the chiral limit. The relation between the axial coupling constant $\tilde{g}$ defined here and the coupling $g$ which is employed usually in the heavy meson ChPT [7-9, 58] is $\tilde{g}=\sqrt{M_{D} M_{D^{*}}} g$. Following ref. [59], we take $g=0.570 \pm 0.006$, 


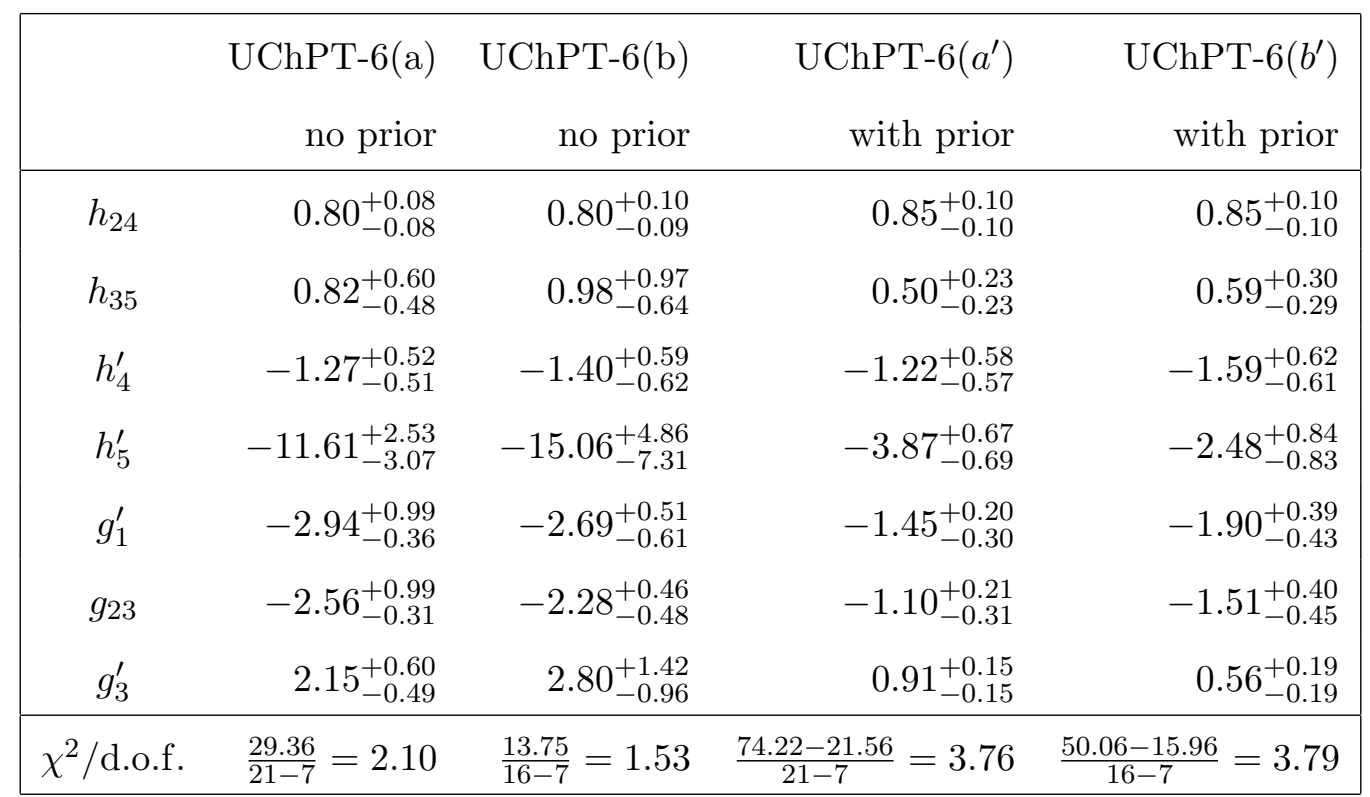

Table 7. Values of the LECs from the 6-channel fits (including explicit $D^{*}$ ) using the method of UChPT. The $h_{i}$ 's are dimensionless, and the $g_{1}^{\prime}, g_{23}$ and $g_{3}^{\prime}$ are in $\mathrm{GeV}^{-1}$.

determined by calculating the decay width of the process $D^{*+} \rightarrow D^{0} \pi^{+}$, and then one gets $\tilde{g} \simeq(1103.3 \pm 11.6) \mathrm{MeV}$. The calculations of the Feynman diagrams in figure 7 are straightforward but the analytical results are too lengthy to be shown here. Similar to eq. (5.2), the pion-mass dependence of the $D^{*}$ and $D_{s}^{*}$ masses reads

$$
M_{D^{*}}=\stackrel{\circ}{M_{D^{*}}}+\left(\tilde{h}_{1}+2 \tilde{h}_{0}\right) \frac{M_{\pi}^{2}}{\stackrel{\circ}{M}_{D^{*}}}, \quad M_{D_{s}{ }^{*}}=\stackrel{\circ}{M}_{D_{s}{ }^{*}}+2 \tilde{h}_{0} \frac{M_{\pi}^{2}}{\stackrel{\circ}{M}_{D_{s}{ }^{*}}},
$$

where $\tilde{h}_{0}$ and $\tilde{h}_{1}$ are the analogues of $h_{0}$ and $h_{1}$, respectively. In the heavy quark limit, one has $\tilde{h}_{1}=h_{1}$ and $\tilde{h}_{0}=h_{0}$. As discussed in ref. [26], the breaking of heavy quark spin symmetry is only about $3 \%$. Therefore, to a good approximation, we impose these two heavy-quark limit relations. The masses of the vector charmed mesons in the limit of $M_{\pi} \rightarrow 0$, i.e. ${\stackrel{\circ}{D^{*}}}$ and $\stackrel{\circ}{M}_{D_{s}^{*}}$, are related to the corresponding ones of the pseudoscalar charmed mesons via

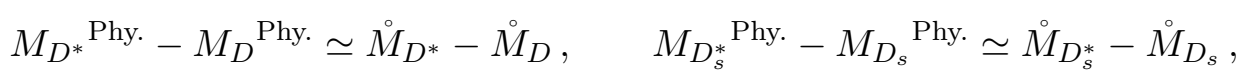

with $M_{D^{*}}^{\text {Phy. }}=2008.6 \mathrm{MeV}$ and $M_{D_{s}^{*}}^{\text {Phy. }}=2112.3 \mathrm{MeV}$, which are physical masses for $D^{*}$ and $D_{s}^{*}$, respectively. In parallel to the four kinds of 6 -channel fits in the previous section, we refit the $S$-wave scattering lengths and the results are shown in table 7 . In each case, the LECs as well as the chi-squared are almost same as before. This implies that the influence of $D^{*}$ to the $S$-wave scattering lengths is marginal and it is a good approximation to exclude them in the calculation. 


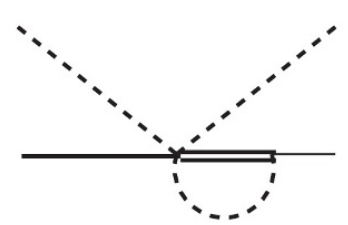

(a)

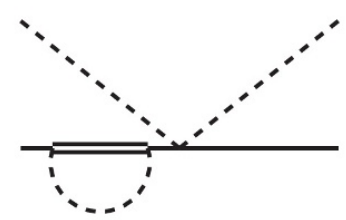

(d)

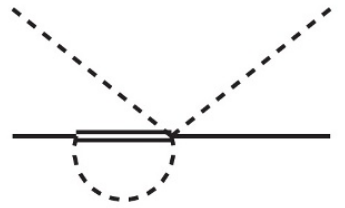

(b)

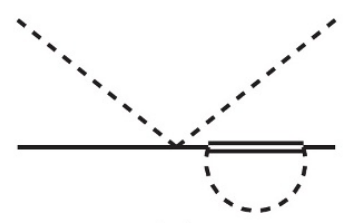

(e)

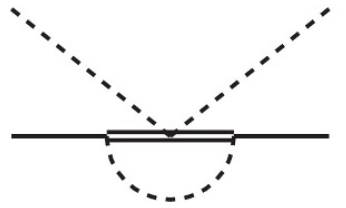

(c)

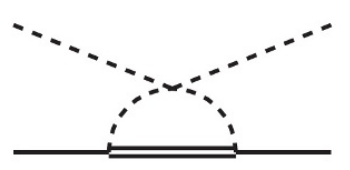

(f)

Figure 7. Feynman diagrams (including the vector charmed mesons) that survive in the heavy quark limit.

\section{Summary and outlook}

We have computed the $D$ - $\phi$ scattering amplitude that is valid up to the NNLO in the chiral expansion within the framework of ChPT. The complete analytical expressions for the amplitudes are given using a renormalization procedure with the EOMS subtraction scheme. We show explicitly that the UV divergences and the PCB terms, both of which stem from the loops, can be absorbed into the LECs. We then obtained the EOMSrenormalized $D$ - $\phi$ scattering amplitudes which are independent of the renormalization scale and possess good properties such as correct power counting and proper analyticity.

In order to describe the lattice data on the $S$-wave scattering lengths at relatively high pion masses and to account for the nonperturbative nature in the channels like the $(S, I)=$ $(1,0) D K$, the aforementioned perturbative amplitudes are inserted into a unitarization procedure to perform the chiral extrapolation from large unphysical light quark masses down to the $\mathrm{SU}(2)$ chiral limit. We tried different fitting procedures with and without a naturalness constraint. It turns out that the absolute value of $h_{5}^{\prime}$ could be quite large if the naturalness constraint is not put by hand. We want to stress that more lattice simulations in different channels are necessary for a better determination of the involved LECs and a better understanding of the scalar and axial-vector charmed mesons. When the LECs are well constrained, we can make reliable predictions in the channels which have not been calculated on the lattice and in the bottom sector utilizing heavy quark spin symmetry.

\section{Acknowledgments}

We would like to thank C. Hanhart and Tom Luu for useful discussions and comments. This work is supported in part by DFG and NSFC through funds provided to the SinoGerman CRC 110 "Symmetries and the Emergence of Structure in QCD" (NSFC Grant No. 11261130311), and by NSFC (Grant No. 11165005). The work of UGM was supported in part by The Chinese Academy of Sciences (CAS) President's International Fellowship Initiative (PIFI) grant no. 2015VMA076. 


\section{A Definition of one-loop integrals}

In this appendix, all the relevant one-loop integrals are defined. For the current case, only one- and two-point loop functions are involved. As is well known, each tensor one-loop integral can be expressed as a linear sum of scalar one-loop integrals by using the method of Passarino-Veltmann (PV) decomposition [60]. Hence, if the explicit expressions of the scalar one-loop integrals are known, the loop amplitudes can be obtained analytically.

Throughout this work, the ultraviolet divergence is contained in the quantity $R$ which is defined by

$$
R=\frac{2}{d-4}+\gamma_{E}-1-\ln (4 \pi),
$$

with $\gamma_{E}$ the Euler constant and $d$ the space-time dimension. In addition, we will denote the renormalization scale by $\mu$. In terms of these notations, various loop integrals involved in the calculations are given as follows:

- One-point loop function:

$$
\mathcal{I}_{a}=\frac{\mu^{4-d}}{i} \int \frac{\mathrm{d}^{d} k}{(2 \pi)^{d}} \frac{1}{k^{2}-M_{a}^{2}+i 0^{+}}=-\frac{M_{a}^{2}}{16 \pi^{2}}\left(R+\ln \frac{M_{a}^{2}}{\mu^{2}}\right) .
$$

- Two-point loop function for unequal masses: $\left(M_{a}>M_{b}\right)$

$$
\begin{aligned}
& \left\{\mathcal{H}_{a b}\left(p^{2}\right), p^{\mu} \mathcal{H}_{a b}^{1}\left(p^{2}\right), g^{\mu \nu} \mathcal{H}_{a b}^{00}\left(p^{2}\right)+p^{\mu} p^{\nu} \mathcal{H}_{a b}^{11}\left(p^{2}\right)\right\} \\
& \quad=\frac{\mu^{4-d}}{i} \int \frac{\mathrm{d}^{d} k}{(2 \pi)^{d}} \frac{\left\{1, k^{\mu}, k^{\mu} k^{\nu}\right\}}{\left(k^{2}-M_{a}^{2}+i 0^{+}\right)\left[(k+p)^{2}-M_{b}^{2}+i 0^{+}\right]},
\end{aligned}
$$

where the PV coefficients are given by

$$
\begin{aligned}
\mathcal{H}_{a b}^{1}\left(p^{2}\right)= & \frac{1}{2 p^{2}}\left[\mathcal{I}_{a}-\mathcal{I}_{b}-\left(p^{2}+\Delta_{a b}\right) \mathcal{H}_{a b}\left(p^{2}\right)\right] \\
\mathcal{H}_{a b}^{00}\left(p^{2}\right)= & \frac{1}{12 p^{2}}\left\{\left(p^{2}+\Delta_{a b}\right) \mathcal{I}_{a}+\left(p^{2}-\Delta_{a b}\right) \mathcal{I}_{b}+\left[4 p^{2} M_{a}^{2}-\left(p^{2}+\Delta_{a b}\right)^{2}\right] \mathcal{H}_{a b}\left(p^{2}\right)\right\} \\
& -\frac{1}{16 \pi^{2}} \frac{1}{18}\left(p^{2}-3 \Sigma_{a b}\right), \\
\mathcal{H}_{a b}^{11}\left(p^{2}\right)= & \frac{1}{3 p^{4}}\left\{-\left(p^{2}+\Delta_{a b}\right) \mathcal{I}_{a}+\left(2 p^{2}+\Delta_{a b}\right) \mathcal{I}_{b}-\left[p^{2} M_{a}^{2}-\left(p^{2}+\Delta_{a b}\right)^{2}\right] \mathcal{H}_{a b}\left(p^{2}\right)\right\} \\
& +\frac{1}{16 \pi^{2}} \frac{1}{18 p^{2}}\left(p^{2}-3 \Sigma_{a b}\right),
\end{aligned}
$$

where we have defined $\Delta_{a b} \equiv M_{a}^{2}-M_{b}^{2}$ and $\Sigma_{a b} \equiv M_{a}^{2}+M_{b}^{2}$. The scalar two-point one-loop function $\mathcal{H}_{a b}\left(p^{2}\right)$ has the following analytical form,

$$
\begin{aligned}
\mathcal{H}_{a b}\left(p^{2}\right)=\frac{1}{16 \pi^{2}}[ & -R+1-\ln \frac{M_{b}^{2}}{\mu^{2}}+\frac{\Delta_{a b}+p^{2}}{2 p^{2}} \ln \frac{M_{b}^{2}}{M_{a}^{2}} \\
& \left.+\frac{p^{2}-\left(M_{a}-M_{b}\right)^{2}}{p^{2}} \rho_{a b}\left(p^{2}\right) \ln \frac{\rho_{a b}\left(p^{2}\right)-1}{\rho_{a b}\left(p^{2}\right)+1}\right],
\end{aligned}
$$


with

$$
\rho_{a b}\left(p^{2}\right) \equiv \sqrt{\frac{p^{2}-\left(M_{a}+M_{b}\right)^{2}}{p^{2}-\left(M_{a}-M_{b}\right)^{2}}} .
$$

To get the imaginary part above the threshold properly, one should take the branch cut for the logarithm along the negative real axis.

- Two-point loop function for equal masses:

$$
\begin{aligned}
& \left\{\mathcal{J}_{a}\left(p^{2}\right), p^{\mu} \mathcal{J}_{a}^{1}\left(p^{2}\right), g^{\mu \nu} \mathcal{J}_{a}^{00}\left(p^{2}\right)+p^{\mu} p^{\nu} \mathcal{J}_{a}^{11}\left(p^{2}\right)\right\} \\
& =\frac{\mu^{4-d}}{i} \int \frac{\mathrm{d}^{d} k}{(2 \pi)^{d}} \frac{\left\{1, k^{\mu}, k^{\mu} k^{\nu}\right\}}{\left(k^{2}-M_{a}^{2}+i 0^{+}\right)\left[(k-p)^{2}-M_{a}^{2}+i 0^{+}\right]},
\end{aligned}
$$

where the Passarino-Veltmann coefficients are given by

$$
\begin{aligned}
\mathcal{J}_{a}^{1}\left(p^{2}\right) & =-\frac{1}{2} \mathcal{J}_{a}\left(p^{2}\right), \\
\mathcal{J}_{a}^{00}\left(p^{2}\right) & =\frac{1}{12}\left(4 M_{a}^{2}-p^{2}\right) \mathcal{J}_{a}\left(p^{2}\right)+\frac{1}{6} \mathcal{I}_{a}+\frac{1}{16 \pi^{2}} \frac{1}{18}\left(6 M_{a}^{2}-p^{2}\right), \\
\mathcal{J}_{a}^{11}\left(p^{2}\right) & =\frac{1}{3 p^{2}}\left[\left(p^{2}-M_{a}^{2}\right) \mathcal{J}_{a}\left(p^{2}\right)+\mathcal{I}_{a}\right]+\frac{1}{16 \pi^{2}} \frac{1}{18 p^{2}}\left(p^{2}-6 M_{a}^{2}\right) .
\end{aligned}
$$

In this case, the scalar two-point one-loop function $\mathcal{J}_{a}\left(p^{2}\right)$ has a much simpler analytical form,

$$
\mathcal{J}_{a}\left(p^{2}\right)=\frac{1}{16 \pi^{2}}\left[-R+1-\ln \frac{M_{a}^{2}}{\mu^{2}}+\sigma_{a}\left(p^{2}\right) \ln \frac{\sigma_{a}\left(p^{2}\right)-1}{\sigma_{a}\left(p^{2}\right)+1}\right], \quad \sigma_{a}\left(p^{2}\right) \equiv \sqrt{1-\frac{4 M_{a}^{2}}{p^{2}}} .
$$

\section{B Loop amplitudes without explicit charmed vector mensons}

In order to express the loop amplitude in a short form, the following abbreviation is adopted,

$$
\begin{aligned}
\mathcal{F}_{a b}^{(c d)}(s, t)= & {\left[3\left(s-M_{a}^{2}\right)+\left(s-M_{c}^{2}\right)\right] \mathcal{I}_{d}-\left(s-\Sigma_{b c}\right)^{2} \mathcal{H}_{c d}(s)+2\left(t-2 M_{b}^{2}\right) \mathcal{H}_{c d}^{00}(s) } \\
& +2\left(s-\Delta_{a b}\right)\left(s-\Sigma_{b c}\right) \mathcal{H}_{c d}^{1}(s)-\left(s-\Delta_{a b}\right)^{2} \mathcal{H}_{c d}^{11}(s) .
\end{aligned}
$$

We first list the loop amplitudes concerning the elastic scattering processes.

- $D^{0} K^{-} \rightarrow D^{0} K^{-}$

$$
\begin{aligned}
\mathcal{A}_{D^{0} K^{-} \rightarrow D^{0} K^{-}}^{\text {lop }}(s, t)= & \frac{1}{16 F^{4}}\left\{\mathcal{F}_{D K}^{(D K)}(s, t)+2 \mathcal{F}_{D K}^{(D K)}(u, t)+\frac{3}{2} \mathcal{F}_{D K}^{\left(D_{s} \eta\right)}(u, t)+\frac{1}{2} \mathcal{F}_{D K}^{\left(D_{s} \pi\right)}(u, t)\right. \\
& \left.+(s-u)\left(\mathcal{I}_{\eta}+2 \mathcal{I}_{K}+\mathcal{I}_{\pi}\right)-4(s-u)\left[\mathcal{J}_{\pi}^{00}(t)+2 \mathcal{J}_{K}^{00}(t)\right]\right\}
\end{aligned}
$$

- $D^{+} K^{+} \rightarrow D^{+} K^{+}$

$$
\mathcal{A}_{D^{+} K^{+} \rightarrow D^{+} K^{+}}^{\text {loop }}(s, t)=\frac{1}{16 F^{4}}\left\{\mathcal{F}_{D K}^{\left(D_{s} \pi\right)}(s, t)+\mathcal{F}_{D K}^{(D K)}(u, t)-4(s-u)\left[\mathcal{J}_{\pi}^{00}(t)-\mathcal{J}_{K}^{00}(t)\right]\right\} .
$$


- $D^{+} \pi^{+} \rightarrow D^{+} \pi^{+}$

$$
\begin{aligned}
\mathcal{A}_{D^{+} \pi^{+} \rightarrow D^{+} \pi^{+}}^{\text {loop }}(s, t)= & \frac{1}{16 F^{4}}\left\{\mathcal{F}_{D \pi}^{(D \pi)}(s, t)+3 \mathcal{F}_{D \pi}^{(D \pi)}(u, t)+\mathcal{F}_{D \pi}^{\left(D_{s} K\right)}(u, t)\right. \\
& \left.+\frac{4}{3}(s-u)\left(2 \mathcal{I}_{\pi}+\mathcal{I}_{K}\right)-4(s-u)\left[2 \mathcal{J}_{\pi}^{00}(t)+\mathcal{J}_{K}^{00}(t)\right]\right\} .
\end{aligned}
$$

- $D^{+} \eta \rightarrow D^{+} \eta$

$$
\mathcal{A}_{D^{+} \eta \rightarrow D^{+} \eta}^{\text {loop }}(s, t)=\frac{1}{16 F^{4}}\left[\frac{3}{2} \mathcal{F}_{D \eta}^{\left(D_{s} K\right)}(s, t)+\frac{3}{2} \mathcal{F}_{D \eta}^{\left(D_{s} K\right)}(u, t)\right] .
$$

- $D_{s}^{+} K^{+} \rightarrow D_{s}^{+} K^{+}$

$$
\begin{aligned}
\mathcal{A}_{D_{s}^{+} K^{+} \rightarrow D_{s}^{+} K^{+}}^{\text {loop }}(s, t)= & \frac{1}{16 F^{4}}\left[\mathcal{F}_{D_{s} K}^{\left(D_{s} K\right)}(s, t)+\mathcal{F}_{D_{s} K}^{\left(D_{s} K\right)}(u, t)+\frac{3}{2} \mathcal{F}_{D_{s} K}^{(D \eta)}(u, t)+\frac{3}{2} \mathcal{F}_{D_{s} K}^{(D \pi)}(u, t)\right. \\
& \left.+(s-u)\left(\mathcal{I}_{\eta}+2 \mathcal{I}_{K}+\mathcal{I}_{\pi}\right)-12(s-u) \mathcal{J}_{K}^{00}(t)\right]
\end{aligned}
$$

- $D_{s}^{+} \eta \rightarrow D_{s}^{+} \eta$

$$
\mathcal{A}_{D_{s}^{+} \eta \rightarrow D_{s}^{+} \eta}^{\text {lop }}(s, t)=\frac{1}{16 F^{4}}\left[3 \mathcal{F}_{D_{s} \eta}^{(D K)}(s, t)+3 \mathcal{F}_{D_{s} \eta}^{(D K)}(u, t)\right] .
$$

- $D_{s}^{+} \pi^{0} \rightarrow D_{s}^{+} \pi^{0}$

$$
\mathcal{A}_{D_{s}^{+} \pi^{0} \rightarrow D_{s}^{+} \pi^{0}}^{\text {lop }}(s, t)=\frac{1}{16 F^{4}}\left[\mathcal{F}_{D_{s} \pi}^{(D K)}(s, t)+\mathcal{F}_{D_{s} \pi}^{(D K)}(u, t)\right] .
$$

As for the inelastic processes, the amplitudes become a little more complicated. To reduce them, we further need

$$
\mathcal{G}_{a b, c d}^{(e f)}(s, t)=\frac{1}{2} \Delta_{b d}^{2} \mathcal{H}_{e f}(s)+\frac{1}{2}\left(\Delta_{a c}-\Delta_{b d}\right)^{2} \mathcal{H}_{e f}^{11}(s)-\Delta_{b d}\left(\Delta_{a c}-\Delta_{b d}\right) \mathcal{H}_{e f}^{1}(s) .
$$

In the above equation, the letters $a$ and $b(c$ and $d$ ) label the incoming (outgoing) particles, while $e$ and $f$ mark the particles in the loop. This convention also holds for the abbreviations ${ }^{1} \mathcal{K}_{a b, c d}^{(e f)}(s, t)$ and ${ }^{2} \mathcal{K}_{a b, c d}^{(e f)}(s, t)$, whose explicit expressions are given by

$$
\begin{aligned}
{ }^{1} \mathcal{K}_{a b, c d}^{(e f)}(s, t)= & \Delta_{a c}\left\{\frac{1}{2} \mathcal{I}_{f}+\frac{1}{2} t\left[\mathcal{H}_{e f}(t)+\mathcal{H}_{e f}^{1}(t)\right]-\mathcal{H}_{e f}^{00}(t)-t \mathcal{H}_{e f}^{11}(t)\right\}, \\
{ }^{2} \mathcal{K}_{a b, c d}^{(e f)}(s, t)= & -3(s-u) \mathcal{H}_{e f}^{00}(t)-\Delta_{a c}\left[\frac{1}{6}\left(6 \Sigma_{d e}+6 \Delta_{d f}-13 \Delta_{b d}\right) \mathcal{H}_{e f}^{1}(t)\right. \\
& \left.+\frac{1}{6}\left(3 \Sigma_{d e}+3 \Delta_{d f}-2 \Delta_{b d}\right) \mathcal{H}_{e f}(t)-3 \Delta_{b d} \mathcal{H}_{e f}^{11}(t)\right] .
\end{aligned}
$$

In combination with the abovementioned notations, the inelastic one-loop scattering amplitudes are given as follows: 
- $D^{0} \eta \rightarrow D^{0} \pi^{0}$

$$
\mathcal{A}_{D^{0} \eta \rightarrow D^{0} \pi^{0}}^{\text {loop }}(s, t)=\frac{\sqrt{3}}{64 F^{4}}\left[\mathcal{F}_{D \eta}^{\left(D_{s} K\right)}(s, t)+\mathcal{F}_{D \pi}^{\left(D_{s} K\right)}(s, t)+2 \mathcal{G}_{D \eta, D \pi}^{\left(D_{s} K\right)}(s, t)+(s \leftrightarrow u)\right] .
$$

- $D_{s}^{+} K^{-} \rightarrow D^{0} \pi^{0}$

$$
\begin{aligned}
\mathcal{A}_{D_{s}^{+} K^{-} \rightarrow D^{0} \pi^{0}}^{\text {loop }}(s, t)= & \frac{\sqrt{2}}{16 F^{4}}\left\{\frac{1}{2}\left[\mathcal{F}_{D_{s} K}^{(D \pi)}(s, t)+\mathcal{F}_{D \pi}^{(D \pi)}(s, t)+2 \mathcal{G}_{D_{s} K, D \pi}^{(D \pi)}(s, t)\right]\right. \\
& +\frac{1}{4}\left[\mathcal{F}_{D_{s} K}^{\left(D_{s} K\right)}(s, t)+\mathcal{F}_{D \pi}^{\left(D_{s} K\right)}(s, t)+2 \mathcal{G}_{D_{s} K, D \pi}^{\left(D_{s} K\right)}(s, t)\right] \\
& -\frac{s-u}{12}\left(3 \mathcal{I}_{\eta}+11 \mathcal{I}_{\pi}+10 \mathcal{I}_{K}\right)+\frac{\Delta_{D_{s} D}}{24}\left(3 \mathcal{I}_{\eta}-5 \mathcal{I}_{\pi}+2 \mathcal{I}_{K}\right) \\
& -\left({ }^{1} \mathcal{K}_{D_{s} K, D \pi}^{(\eta K)}(s, t)+{ }^{2} \mathcal{K}_{D_{s} K, D \pi}^{(\eta K)}(s, t)\right) \\
& \left.-\left(\frac{5}{3}{ }^{1} \mathcal{K}_{D_{s} K, D \pi}^{(K \pi)}(s, t)+{ }^{2} \mathcal{K}_{D_{s} K, D \pi}^{(K \pi)}(s, t)\right)\right\}
\end{aligned}
$$

- $D_{s}^{+} K^{-} \rightarrow D^{0} \eta$

$$
\begin{aligned}
\mathcal{A}_{D_{s}^{+} K^{-} \rightarrow D^{0} \eta}^{\text {loop }}(s, t)= & \frac{\sqrt{6}}{16 F^{4}}\left\{\frac{1}{4}\left(\mathcal{F}_{D_{s} K}^{\left(D_{s} K\right)}(s, t)+\mathcal{F}_{D \eta}^{\left(D_{s} K\right)}(s, t)+2 \mathcal{G}_{D_{s} K, D \eta}^{\left(D_{s} K\right)}(s, t)\right)\right. \\
& -\frac{1}{2}\left(\mathcal{F}_{D_{s} K}^{(D K)}(u, t)+\mathcal{F}_{D \eta}^{(D K)}(u, t)+\mathcal{G}_{D_{s} K, D \eta}^{(D K)}(u, t)+\mathcal{G}_{D_{s} \eta, D K}^{(D K)}(u, t)\right) \\
& +\left({ }^{1} \mathcal{K}_{D_{s} K, D \eta}^{(K \pi)}(s, t)-{ }^{2} \mathcal{K}_{D_{s} K, D \eta}^{(K \pi)}(s, t)\right) \\
& -\left({ }^{1} \mathcal{K}_{D_{s} K, D \eta}^{(\eta K)}(s, t)+{ }^{2} \mathcal{K}_{D_{s} K, D \eta}^{(\eta K)}(s, t)\right) \\
& +\frac{\Delta_{D_{s} D}}{6}\left(5 M_{\eta}^{2}+8 M_{K}^{2}-M_{\pi}^{2}\right)\left(2 \mathcal{H}_{K \pi}^{1}(t)+\mathcal{H}_{K \pi}(t)\right) \\
& -\frac{\Delta_{D_{s} D}}{3}\left(M_{\eta}^{2}-4 M_{K}^{2}+M_{\pi}^{2}\right)\left(2 \mathcal{H}_{\eta K}^{1}(t)+\mathcal{H}_{\eta K}(t)\right) \\
& \left.+\frac{\Delta_{D_{s} D}}{8}\left(\mathcal{I}_{\eta}+\mathcal{I}_{\pi}-2 \mathcal{I}_{K}\right)-\frac{s-u}{4}\left(\mathcal{I}_{\eta}+\mathcal{I}_{\pi}+6 \mathcal{I}_{K}\right)\right\}
\end{aligned}
$$

\section{Infrared regular parts of the loop integrals}

The following expressions for the infrared regular parts are taken from ref. [61] with the nucleon mass (pion) mass) replaced by the $D$ meson (Goldstone boson) mass:

- one-point: $a \in\left\{D, D_{s}\right\}$

$$
\mathcal{I}_{a}^{\text {reg. }}=-\frac{M_{a}^{2}}{16 \pi^{2}} \ln \frac{M_{a}^{2}}{\mu^{2}} .
$$

- two-point: $a \in\left\{D, D_{s}\right\}$ and $b \in\{\pi, K, \eta\}$

$$
\begin{aligned}
\mathcal{H}_{a b}^{\text {reg. }}(s)= & \frac{1}{16 \pi^{2}}\left(1-\log \frac{M_{a}^{2}}{\mu^{2}}\right)-\frac{s-M_{a}^{2}}{2 M_{a}^{2}} \frac{1}{16 \pi^{2}}\left(1-\log \frac{M_{a}^{2}}{\mu^{2}}\right) \\
& +\frac{1}{32 \pi^{2}}\left[\frac{M_{b}^{2}}{M_{a}^{2}}\left(3+\log \frac{M_{a}^{2}}{\mu^{2}}\right)-\left(\frac{s-M_{a}^{2}}{M_{a}^{2}}\right)^{2} \log \frac{M_{a}^{2}}{\mu^{2}}\right]+\mathcal{O}\left(p^{3}\right) .
\end{aligned}
$$


The power counting breaking term of $\mathcal{F}_{a b}^{(c d)}(s, t)$ is of $\mathcal{O}\left(p^{2}\right)$ and its explicit form reads

$$
\begin{aligned}
\mathcal{F}_{a b}^{(c d)}(s, t)^{\mathrm{PCB}}= & \frac{1}{16 \pi^{2}}\left\{2\left(s-M_{a}^{2}\right)\left(s-M_{c}^{2}\right)\left[\frac{1}{2} \log \frac{M_{c}^{2}}{\mu^{2}}-1\right]-\left(s-M_{a}^{2}\right)^{2}\left[\frac{8}{9}-\frac{1}{3} \log \frac{M_{c}^{2}}{\mu^{2}}\right]\right. \\
& \left.-\left(s-M_{c}\right)^{2}\left[1-\log \frac{M_{c}^{2}}{\mu^{2}}\right]+2\left(t-2 M_{b}^{2}\right) M_{c}^{2}\left[\frac{1}{9}-\frac{1}{6} \log \frac{M_{c}^{2}}{\mu^{2}}\right]\right\} .
\end{aligned}
$$

Since the difference between $M_{a}^{2}$ and $M_{c}^{2}$ is at least $\mathcal{O}\left(p^{2}\right)$, the above expression can be reduced to a simpler form

$$
\begin{aligned}
& \mathcal{F}_{a b}^{(c d)}(s, t)^{\mathrm{PCB}}=\frac{1}{144 \pi^{2}}\{ {\left[2\left(t-2 M_{b}^{2}\right) M_{c}^{2}-35\left(s-M_{c}^{2}\right)^{2}\right] } \\
&\left.+3\left[7\left(s-M_{c}^{2}\right)^{2}-\left(t-2 M_{b}^{2}\right) M_{c}^{2}\right] \log \frac{M_{c}^{2}}{\mu^{2}}\right\} .
\end{aligned}
$$

Open Access. This article is distributed under the terms of the Creative Commons Attribution License (CC-BY 4.0), which permits any use, distribution and reproduction in any medium, provided the original author(s) and source are credited.

\section{References}

[1] BABAR collaboration, B. Aubert et al., Observation of a narrow meson decaying to $D_{s}^{+} \pi^{0}$ at a mass of $2.32 \mathrm{GeV} / \mathrm{c}^{2}$, Phys. Rev. Lett. 90 (2003) 242001 [hep-ex/0304021] [INSPIRE].

[2] Belle collaboration, P. Krokovny et al., Observation of the $D_{s J}(2317)$ and $D_{s J}(2457)$ in $B$ decays, Phys. Rev. Lett. 91 (2003) 262002 [hep-ex/0308019] [InSPIRE].

[3] T. Barnes, F.E. Close and H.J. Lipkin, Implications of a DK molecule at $2.32 \mathrm{GeV}$, Phys. Rev. D 68 (2003) 054006 [hep-ph/0305025] [INSPIRE].

[4] E.E. Kolomeitsev and M.F.M. Lutz, On heavy light meson resonances and chiral symmetry, Phys. Lett. B 582 (2004) 39 [hep-ph/0307133] [INSPIRE].

[5] F.-K. Guo, P.-N. Shen, H.-C. Chiang, R.-G. Ping and B.-S. Zou, Dynamically generated $0^{+}$ heavy mesons in a heavy chiral unitary approach, Phys. Lett. B 641 (2006) 278 [hep-ph/0603072] [INSPIRE].

[6] D. Gamermann, E. Oset, D. Strottman and M.J. Vicente Vacas, Dynamically generated open and hidden charm meson systems, Phys. Rev. D 76 (2007) 074016 [hep-ph/0612179] [INSPIRE].

[7] G. Burdman and J.F. Donoghue, Union of chiral and heavy quark symmetries, Phys. Lett. B 280 (1992) 287 [INSPIRE].

[8] M.B. Wise, Chiral perturbation theory for hadrons containing a heavy quark, Phys. Rev. D 45 (1992) 2188 [INSPIRE].

[9] T.-M. Yan, H.-Y. Cheng, C.-Y. Cheung, G.-L. Lin, Y.C. Lin and H.-L. Yu, Heavy quark symmetry and chiral dynamics, Phys. Rev. D 46 (1992) 1148 [Erratum ibid. D 55 (1997) 5851] [INSPIRE].

[10] J. Hofmann and M.F.M. Lutz, Open charm meson resonances with negative strangeness, Nucl. Phys. A 733 (2004) 142 [hep-ph/0308263] [inSPIRE]. 
[11] F.-K. Guo, C. Hanhart, S. Krewald and U.-G. Meißner, Subleading contributions to the width of the $D_{s 0}^{*}(2317)$, Phys. Lett. B 666 (2008) 251 [arXiv:0806.3374] [INSPIRE].

[12] F.-K. Guo, C. Hanhart and U.-G. Meißner, Interactions between heavy mesons and Goldstone bosons from chiral dynamics, Eur. Phys. J. A 40 (2009) 171 [arXiv:0901.1597] [InSPIRE].

[13] M. Cleven, F.-K. Guo, C. Hanhart and U.-G. Meißner, Light meson mass dependence of the positive parity heavy-strange mesons, Eur. Phys. J. A 47 (2011) 19 [arXiv:1009.3804] [INSPIRE].

[14] Z.-H. Guo, U.-G. Meißner and D.-L. Yao, New insights into the $D_{s 0}^{*}(2317)$ and other charm scalar mesons, Phys. Rev. D 92 (2015) 094008 [arXiv: 1507.03123] [InSPIRE].

[15] L. Liu, H.-W. Lin and K. Orginos, Charmed hadron interactions, PoS(LATTICE 2008)112 [arXiv:0810.5412] [INSPIRE].

[16] L. Liu, K. Orginos, F.-K. Guo, C. Hanhart and U.-G. Meißner, Interactions of charmed mesons with light pseudoscalar mesons from lattice $Q C D$ and implications on the nature of the $D_{s 0}^{*}(2317)$, Phys. Rev. D 87 (2013) 014508 [arXiv: 1208.4535] [InSPIRE].

[17] D. Mohler, S. Prelovsek and R.M. Woloshyn, D $\pi$ scattering and $D$ meson resonances from lattice QCD, Phys. Rev. D 87 (2013) 034501 [arXiv: 1208.4059] [InSPIRE].

[18] D. Mohler, C.B. Lang, L. Leskovec, S. Prelovsek and R.M. Woloshyn, $D_{s 0}^{*}(2317)$ meson and D-meson-kaon scattering from lattice QCD, Phys. Rev. Lett. 111 (2013) 222001 [arXiv: 1308.3175] [INSPIRE].

[19] C.B. Lang, L. Leskovec, D. Mohler, S. Prelovsek and R.M. Woloshyn, $D_{s}$ mesons with DK and $D^{*} K$ scattering near threshold, Phys. Rev. D 90 (2014) 034510 [arXiv:1403.8103] [INSPIRE].

[20] A. Martínez Torres, E. Oset, S. Prelovsek and A. Ramos, Reanalysis of lattice QCD spectra leading to the $D_{s 0}^{*}(2317)$ and $D_{s 1}^{*}(2460)$, JHEP 05 (2015) 153 [arXiv:1412.1706] [INSPIRE].

[21] D. Agadjanov, F.K. Guo, G. Ríos and A. Rusetsky, Bound states on the lattice with partially twisted boundary conditions, JHEP 01 (2015) 118 [arXiv:1411.1859] [INSPIRE].

[22] A. Martínez Torres, L.R. Dai, C. Koren, D. Jido and E. Oset, The KD, $\eta D_{s}$ interaction in finite volume and the nature of the $D_{s^{*} 0}(2317)$ resonance, Phys. Rev. D 85 (2012) 014027 [arXiv: 1109.0396] [INSPIRE].

[23] Y.-R. Liu, X. Liu and S.-L. Zhu, Light pseudoscalar meson and heavy meson scattering lengths, Phys. Rev. D 79 (2009) 094026 [arXiv:0904.1770] [INSPIRE].

[24] L.-S. Geng, N. Kaiser, J. Martin-Camalich and W. Weise, Low-energy interactions of Nambu-Goldstone bosons with D mesons in covariant chiral perturbation theory, Phys. Rev. D 82 (2010) 054022 [arXiv: 1008.0383] [INSPIRE].

[25] P. Wang and X.-G. Wang, Study on $0^{+}$states with open charm in unitarized heavy meson chiral approach, Phys. Rev. D 86 (2012) 014030 [arXiv:1204.5553] [INSPIRE].

[26] M. Altenbuchinger, L.-S. Geng and W. Weise, Scattering lengths of Nambu-Goldstone bosons off D mesons and dynamically generated heavy-light mesons, Phys. Rev. D 89 (2014) 014026 [arXiv: 1309.4743] [INSPIRE].

[27] M. Altenbuchinger and L.-S. Geng, Off-shell effects on the interaction of Nambu-Goldstone bosons and D mesons, Phys. Rev. D 89 (2014) 054008 [arXiv:1310.5224] [InSPIRE]. 
[28] J.A. Oller and E. Oset, Chiral symmetry amplitudes in the $S$ wave isoscalar and isovector channels and the $\sigma, f_{0}(980), a_{0}(980)$ scalar mesons, Nucl. Phys. A 620 (1997) 438 [Erratum ibid. A 652 (1999) 407] [hep-ph/9702314] [INSPIRE].

[29] J.A. Oller and U.-G. Meißner, Chiral dynamics in the presence of bound states: kaon nucleon interactions revisited, Phys. Lett. B 500 (2001) 263 [hep-ph/0011146] [INSPIRE].

[30] S. Weinberg, Phenomenological Lagrangians, Physica A 96 (1979) 327 [InSPIRE].

[31] J. Gasser and H. Leutwyler, Chiral perturbation theory to one loop, Annals Phys. 158 (1984) 142 [INSPIRE].

[32] J. Gasser and H. Leutwyler, Chiral perturbation theory: expansions in the mass of the strange quark, Nucl. Phys. B 250 (1985) 465 [inSPIRE].

[33] J. Gasser, M.E. Sainio and A. Svarc, Nucleons with chiral loops, Nucl. Phys. B 307 (1988) 779 [INSPIRE].

[34] E.E. Jenkins and A.V. Manohar, Baryon chiral perturbation theory using a heavy fermion Lagrangian, Phys. Lett. B 255 (1991) 558 [INSPIRE].

[35] V. Bernard, N. Kaiser, J. Kambor and U.-G. Meißner, Chiral structure of the nucleon, Nucl. Phys. B 388 (1992) 315 [inSPIRE].

[36] T. Becher and H. Leutwyler, Baryon chiral perturbation theory in manifestly Lorentz invariant form, Eur. Phys. J. C 9 (1999) 643 [hep-ph/9901384] [INSPIRE].

[37] T. Fuchs, J. Gegelia, G. Japaridze and S. Scherer, Renormalization of relativistic baryon chiral perturbation theory and power counting, Phys. Rev. D 68 (2003) 056005 [hep-ph/0302117] [INSPIRE].

[38] V. Bernard, Chiral perturbation theory and baryon properties, Prog. Part. Nucl. Phys. 60 (2008) 82 [arXiv: 0706.0312] [INSPIRE].

[39] V. Bernard, N. Kaiser, U.-G. Meißner and A. Schmidt, Aspects of nucleon Compton scattering, Z. Phys. A 348 (1994) 317 [hep-ph/9311354] [INSPIRE].

[40] T.N. Truong, Chiral perturbation theory and final state theorem, Phys. Rev. Lett. 61 (1988) 2526 [INSPIRE].

[41] T.N. Truong, Remarks on the unitarization methods, Phys. Rev. Lett. 67 (1991) 2260 [INSPIRE].

[42] A. Dobado, M.J. Herrero and T.N. Truong, Unitarized chiral perturbation theory for elastic pion-pion scattering, Phys. Lett. B 235 (1990) 134 [INSPIRE].

[43] A. Dobado and J.R. Peláez, A global fit of $\pi \pi$ and $\pi K$ elastic scattering in ChPT with dispersion relations, Phys. Rev. D 47 (1993) 4883 [hep-ph/9301276] [INSPIRE].

[44] A. Dobado and J.R. Peláez, The inverse amplitude method in chiral perturbation theory, Phys. Rev. D 56 (1997) 3057 [hep-ph/9604416] [INSPIRE].

[45] N. Fettes, U.-G. Meißner, M. Mojzis and S. Steininger, The chiral effective pion nucleon Lagrangian of order $p^{4}$, Annals Phys. 283 (2000) 273 [Erratum ibid. 288 (2001) 249] [hep-ph/0001308] [INSPIRE].

[46] T. Hannah, The anomalous process $\gamma \pi \rightarrow \pi \pi$ to two loops, Nucl. Phys. B 593 (2001) 577 [hep-ph/0102213] [INSPIRE]. 
[47] J.A. Oller, E. Oset and J.R. Peláez, Meson meson interaction in a nonperturbative chiral approach, Phys. Rev. D 59 (1999) 074001 [Erratum ibid. D 60 (1999) 099906] [Erratum ibid. D 75 (2007) 099903] [hep-ph/9804209] [INSPIRE].

[48] A. Walker-Loud et al., Light hadron spectroscopy using domain wall valence quarks on an Asqtad sea, Phys. Rev. D 79 (2009) 054502 [arXiv:0806.4549] [INSPIRE].

[49] MILC collaboration, A. Bazavov et al., MILC results for light pseudoscalars, PoS (CD09) 007 [arXiv:0910.2966] [INSPIRE].

[50] R.J. Dowdall, C.T.H. Davies, G.P. Lepage and C. McNeile, $V_{u s}$ from $\pi$ and $K$ decay constants in full lattice QCD with physical $u, d, s$ and c quarks, Phys. Rev. D 88 (2013) 074504 [arXiv: 1303.1670] [INSPIRE].

[51] J. Bijnens and G. Ecker, Mesonic low-energy constants, Ann. Rev. Nucl. Part. Sci. 64 (2014) 149 [arXiv: 1405.6488] [INSPIRE].

[52] F.-K. Guo, L. Liu, U.-G. Meißner and P. Wang, Tetraquarks, hadronic molecules, meson-meson scattering and disconnected contributions in lattice QCD, Phys. Rev. D 88 (2013) 074506 [arXiv: 1308.2545] [INSPIRE].

[53] O. Bär, G. Rupak and N. Shoresh, Chiral perturbation theory at $O\left(a^{2}\right)$ for lattice $Q C D$, Phys. Rev. D 70 (2004) 034508 [hep-lat/0306021] [INSPIRE].

[54] O. Bär, C. Bernard, G. Rupak and N. Shoresh, Chiral perturbation theory for staggered sea quarks and Ginsparg-Wilson valence quarks, Phys. Rev. D 72 (2005) 054502 [hep-lat/0503009] [INSPIRE].

[55] B.C. Tiburzi, Baryons with Ginsparg-Wilson quarks in a staggered sea, Phys. Rev. D 72 (2005) 094501 [Erratum ibid. D 79 (2009) 039904] [hep-lat/0508019] [INSPIRE].

[56] J.-W. Chen, D. O'Connell and A. Walker-Loud, Universality of mixed action extrapolation formulae, JHEP 04 (2009) 090 [arXiv:0706.0035] [INSPIRE].

[57] M.R. Schindler and D.R. Phillips, Bayesian methods for parameter estimation in effective field theories, Annals Phys. 324 (2009) 682 [Erratum ibid. 324 (2009) 2051] [arXiv:0808.3643] [INSPIRE].

[58] B. Grinstein, E.E. Jenkins, A.V. Manohar, M.J. Savage and M.B. Wise, Chiral perturbation theory for $f_{D_{s}} / F_{D}$ and $B_{B_{s}} / B_{B}$, Nucl. Phys. B 380 (1992) 369 [hep-ph/9204207] [INSPIRE].

[59] M. Albaladejo, F.-K. Guo, C. Hidalgo-Duque, J. Nieves and M.P. Valderrama, Decay widths of the spin-2 partners of the X(3872), Eur. Phys. J. C in press [arXiv:1504.00861] [INSPIRE].

[60] G. Passarino and M.J.G. Veltman, One loop corrections for $e^{+} e^{-}$annihilation into $\mu^{+} \mu^{-}$in the Weinberg model, Nucl. Phys. B 160 (1979) 151 [inSPIRE].

[61] Y.-H. Chen, D.-L. Yao and H.-Q. Zheng, Analyses of pion-nucleon elastic scattering amplitudes up to $O\left(p^{4}\right)$ in extended-on-mass-shell subtraction scheme, Phys. Rev. D 87 (2013) 054019 [arXiv:1212.1893] [INSPIRE]. 